\title{
Support Effects on Hydrogen Desorption, Isotope Exchange, Chemical Reactivity and Magnetism of Platinum Nanoclusters in KL Zeolite
}

\author{
Christopher Jensen, ${ }^{1}$ Joris van Slageren, ${ }^{1}$ Peter Jakes, ${ }^{2}$ Rüdiger-A. Eichel, ${ }^{2}$ and Emil \\ Roduner*1,3 \\ ${ }^{1}$ Institut für Physikalische Chemie, Universität Stuttgart, Pfaffenwaldring 55, D-70569 Stuttgart, Germany \\ ${ }^{2}$ Forschungszentrum Jülich, Institut für Energie- und Klimaforschung IEK-9, 52425 Jülich, Germany \\ ${ }^{3}$ Department of Chemistry, University of Pretoria, Pretoria 0002, Republic of South Africa
}

\begin{abstract}
Platinum clusters were prepared by ion exchange of a KL zeolite, followed by oxygen calcination and hydrogen reduction, and characterized by electron paramagnetic resonance (EPR) and hyperfine sublevel correlation experiment (HYSCORE) spectroscopy. Simulations indicate that the cluster contains 12 equivalent platinum atoms. Therefore, the most likely structure is an icosahedral or cuboctahedral magic number $\mathrm{Pt}_{13}$ cluster with 12 platinum atoms at the surface. One atom in the center possesses only a small spin density. H/D desorption and readsorption experiments monitored via EPR and HYSCORE measurements provide information about the structure of the clusters and about the reversibility of the adsorption/desorption and isotope exchange process. Deuterium desorption experiments result in a value of $2.1 \pm 0.2 \mathrm{eV}$ for the $\mathrm{D}_{2}$ desorption energy of the $\mathrm{Pt}_{13}$ clusters dispersed in $\mathrm{KL}$ zeolite. This is more than double of the
\end{abstract}


value for a (111) Pt single-crystal surface, revealing a finite size and/or support effect. Oxygen adsorption on deuterium covered $\mathrm{Pt}_{13}$ clusters did not show a vigorous oxyhydrogen reaction. It appears that the reaction is inhibited by the deuterium coverage.

Keywords: Platinum nanoclusters, EPR spectroscopy, catalytic support effect, magnetic properties.

\section{Introduction}

Metal clusters are of high scientific interest because they are intermediates between atoms and bulk solids. Their physical and chemical properties often differ from those of bulk materials due to the fact that the fraction of surface atoms is large compared to the total number of atoms and the density of states is low. For example, they show enhanced activity and selectivity in heterogeneous catalysis, and they were therefore extensively investigated and obtained high economic relevance. Small platinum clusters dispersed in KL zeolite, for example, are highly active catalysts for important petrochemical reactions like hydrogenation of aromatics and isomerizations.

Clusters in zeolites are commonly prepared by aqueous ion exchange to introduce noble metal ions into the zeolite pores and cages, followed by oxygen calcination and hydrogen reduction. ${ }^{1}$ Details of the preparation procedure are important because they have a great influence on the particle size, and there is a trend of increasing catalytic activity with decreasing cluster size. ${ }^{1,2}$ The distribution of the cluster size is often not uniform, but so-called magic numbers corresponding to a maximum coordination of cluster atoms result in various close-packed polyhedral structures which are energetically preferred relative to others. ${ }^{2}$ In the case of platinum, 
$\mathrm{Pt}_{13}$ is one of the smallest magic number clusters in which 12 equivalent $\mathrm{Pt}$ nuclei on an icosahedron or cuboctahedron surface surround a central Pt atom. Hydrogen on metal cluster surfaces is of great interest and has been investigated extensively because of its basic relevance for understanding elementary steps of catalytic reactions. Hydrogen chemisorption modifies the electronic state of the metal cluster, which causes a change in its catalytic behavior and properties. Furthermore hydrogen chemisorption is often used to determine the dispersion, defined as the fraction of surface atoms of metal catalyst particles. Generally, the chemisorption stoichiometry is assumed to correspond to a single hydrogen atom per surface platinum atom. However, recent work demonstrated, ${ }^{3}$ in agreement with theoretical predictions, ${ }^{4,5}$ that $\mathrm{Pt}_{13}$ clusters can chemisorb $38 \pm 2 \mathrm{H}$ atoms $(\sim 2.9 \pm 0.2 \mathrm{H}$ atoms per $\mathrm{Pt})$ which resist a subsequent evacuation at $308 \mathrm{~K}$.

Watari and Ohnishi studied the electronic structure of $\mathrm{Pt}_{13}$, including hydrogen adsorption, using density functional calculations. ${ }^{6,7}$ They concluded that these clusters have an electronic shell structure, a concept that had been introduced and reviewed previously to describe small metal clusters. ${ }^{8}$ Because the electronic wave functions reflect the near-spherical symmetry of the cluster they resemble atomic orbitals. Therefore, it is a useful concept to regard these clusters as analogs of atoms, called "giant atoms" or "superatoms". ${ }^{9}$ The $5 \mathrm{~d}^{9} 6 \mathrm{~s}^{1}$ valence electrons of Pt occupy states with s, p, d, f, g, and h character, corresponding to cluster molecular orbitals with angular momenta $\ell=0,1,2,3,4$, and 5 . For ideal spherical symmetry these orbitals are energetically $(2 \ell+1)$-fold degenerate, but for the more realistic icosahedral or cuboctahedral cluster structures the degeneracy is partly lifted. Thus, the highest rotation axis of icosahedrons is five-fold, so the maximum orbital degeneracy is five, while in a cuboctahedron it is three. However, spin-orbit coupling or perturbations by the environment (symmetry lowering) lift these degeneracies, which results in irregular progressions of non-degenerate orbitals with average energetic splittings of 1 
eV or less. ${ }^{7}$ If these splittings are less than the spin pairing energy the ground state will nevertheless possess a high spin. Indeed, it was found that a fraction of the $\mathrm{Pt}_{13}$ clusters in $\mathrm{Y}$ zeolite are paramagnetic with magnetic moments of up to $0.65 \mu_{\mathrm{B}}$ per atom. ${ }^{10,11}$ Each $\mathrm{H}$ atom that binds to the cluster pins one of the $\mathrm{Pt}$ valence electrons and engages it in a $\mathrm{Pt}_{\mathrm{n}}-\mathrm{H}$ bond with an energy well below that of the highest occupied state, resulting in a hydride-like character of $\mathrm{H}$. Thus, successive hydrogen adsorption of up to 38 atoms effectively titrates out 38 electrons, starting from the top of the occupied molecular cluster orbitals of the bare $\mathrm{Pt}_{13}$ cluster. Because of the complex energetic sequence of the orbitals this leads, as a function of $\mathrm{H}$ coverage, to a variation of the spin state that is non-trivial to predict.

More recently, it was found that, at room temperature, where the Curie paramagnetism of these clusters is less significant, strong diamagnetism (a factor 35-50 higher than expected for diamagnetic $\mathrm{Pt}$ ) dominates the picture. ${ }^{12}$ This superdiamagnetism is again a consequence of the superatom nature of the cluster in which the diamagnetic ring currents of the many paired valence electrons, including those of the hydride-like chemisorbed hydrogen, extend over the entire cluster, thereby providing a much larger induced magnetic moment antiparallel to the applied magnetic field.

Electron paramagnetic resonance spectroscopy is a powerful method to characterize and investigate small paramagnetic clusters and ions in zeolites and obtain important information of the clusters like size, electronic and geometrical structure, and local environment. Furthermore, EPR is a suitable technique to monitor the development of hydrogen chemisorption on paramagnetic metal cluster surfaces. Schmauke et al. ${ }^{13}$ and Liu et $a l .{ }^{14}$ have reported previously on well-defined $\mathrm{Pt}_{13}$ clusters supported on $\mathrm{KL}$ and $\mathrm{NaY}$ zeolite. 
The aim of the present work is a better understanding of the influence of the difference of the KL versus the NaY zeolite framework on the properties of the Pt clusters. Hence, the results reported in this work should be used for a comparative and complementary discussion with the perceptions of Schmauke and Liu. For the interpretation, it should be kept in mind that any conclusions relate primarily to the EPR active clusters, which are a minority species and represent $<1 \%$ of the entire $\mathrm{Pt}$ in the sample. However, these species are in equilibrium with the EPR inactive diamagnetic and high-spin clusters of very similar size and behavior, as shown by analysis of less selective bulk methods like Extended X-ray Absorption Spectroscopy (EXAFS), quantitative hydrogen chemisorption and SQUID magnetization experiments. ${ }^{11}$

\section{Experimental Methods}

Sample Preparation. KL zeolite was obtained from Chemie Uetikon AG (CU) in Switzerland, and iron-free KL zeolite was obtained from Süd Chemie AG (SC) in Germany. The KL zeolites were first calcined in air using a heating rate of $1 \mathrm{~K} \mathrm{~min}^{-1}$ and holding a final temperature of $773 \mathrm{~K}$ for $14 \mathrm{~h}$ to burn off any organic impurities. $5 \mathrm{~g}$ KL zeolite were stirred in $125 \mathrm{ml}$ of $1 \mathrm{M}$ aqueous $\mathrm{KCl}$ solution at room temperature (RT) for $24 \mathrm{~h}$ to exchange any ions other than $\mathrm{K}^{+}$. The zeolite was filtered off, washed with doubly distilled water to remove $\mathrm{Cl}^{-}$ions, and dried in an oven at $353 \mathrm{~K}$ overnight. This step was repeated three times. Chemical analysis using $\mathrm{ICP}-\mathrm{OES}$ resulted in $\mathrm{Si} / \mathrm{Al}=3.0$ and $\mathrm{K} / \mathrm{Al}=0.98 \mathrm{KL}(\mathrm{CU})$ zeolite, and $\mathrm{Si} / \mathrm{Al}=2.8$ and $\mathrm{K} / \mathrm{Al}$ $=0.98 \mathrm{KL}(\mathrm{SC})$ zeolite.

$\mathrm{Pt} / \mathrm{KL}$ zeolites were prepared by aqueous ion exchange with $109 \mathrm{ml} 3 \mathrm{mM}$ solution of $\left[\mathrm{Pt}\left(\mathrm{NH}_{3}\right)_{4}\right] \mathrm{Cl}_{2}$ (Aldrich) that was added dropwise to a flask containing a suspension of $1 \mathrm{~g} \mathrm{KL}$ zeolite in $500 \mathrm{ml}$ of water. The suspension was stirred for at least 3 days at $343 \mathrm{~K}$. This procedure 
led to a Pt content of $5.3 \mathrm{wt} . \%(\mathrm{CU})$ and $5.5 \mathrm{wt} . \%$ (SC), and for both zeolites to a $\mathrm{K} / \mathrm{Al}$ ratio of $\sim 0.72$, determined by ICP-OES, showing that $26 \%$ of the $\mathrm{K}^{+}$had been exchanged.

Calcination was carried out in flowing $\mathrm{O}_{2}\left(120 \mathrm{ml} \mathrm{min}^{-1}\right)$ using a heating rate of $0.5 \mathrm{~K}$ $\mathrm{min}^{-1}$ from RT to $623 \mathrm{~K}$ and holding this temperature for $5 \mathrm{~h}$. This low heating rate suppresses autoreduction, ${ }^{1}$ drives out the ammonia and leaves the platinum as $\mathrm{Pt}^{2+}(90 \%)$ but in significant amounts also as $\mathrm{Pt}^{+}$and $\mathrm{Pt}^{3+}$ (together 10\%, as determined by EPR). After the sample had cooled to $\mathrm{RT}$, residual $\mathrm{O}_{2}$ in the reactor was purged with $\mathrm{N}_{2}$. Reduction was then performed in flowing $\mathrm{H}_{2}\left(80 \mathrm{ml} \mathrm{min}^{-1}\right)$ at a heating rate of $4 \mathrm{~K} \mathrm{~min}^{-1}$ from RT to $503 \mathrm{~K}$ and keeping the sample at 503 $\mathrm{K}$ for $1 \mathrm{~h}$. The first part of this treatment is effectively the reduction to $\mathrm{Pt}^{0}$, e.g. according to

$$
\begin{array}{llll}
\mathrm{Pt}^{2+}+\mathrm{H}_{2} & \rightarrow & \mathrm{Pt}^{0} & +2 \mathrm{H}^{+}(\mathrm{Z}) \\
(\mathrm{PtOH})^{+}+\mathrm{H}_{2} & \rightarrow & \mathrm{Pt}^{0} & +\mathrm{H}_{2} \mathrm{O}+\mathrm{H}^{+}(\mathrm{Z}) \\
\mathrm{PtO} & +\mathrm{H}_{2} & \rightarrow & \mathrm{Pt}^{0}+\mathrm{H}_{2} \mathrm{O}
\end{array}
$$

where $\mathrm{H}^{+}(\mathrm{Z})$ denotes a Brønsted acid center. Reduction leaves no EPR active paramagnetic Pt ions and is followed by dissociative hydrogen chemisorption:

$$
\mathrm{nPt}^{0}+\mathrm{mH}_{2} \rightarrow \mathrm{Pt}_{\mathrm{n}} \mathrm{H}_{2 \mathrm{~m}} .
$$

Since it is expected that the neutral $\mathrm{Pt}^{0}$ species are much more mobile than their positively charged precursors because of their lower electrostatic interaction with the negatively charged zeolite framework it is assumed that cluster formation occurs after reduction. $\mathrm{Pt}_{13}$ is a "magic" cluster with completed geometric shell which has some extra stability so that this cluster size is favored over others. ${ }^{9}$ For isolated Pt atoms but not for clusters (which is essential for the work carried out below), reduction according to (1a) is reversible. ${ }^{1}$ After cooling to RT in a hydrogen atmosphere, the reactor was purged with $\mathrm{N}_{2}$, and the sample was transferred into an EPR tube in a glove box under nitrogen atmosphere, and then evacuated for EPR experiments. 
For hydrogen/deuterium desorption experiments, an EPR tube containing about $160 \mathrm{mg}$ of $\mathrm{Pt} / \mathrm{KL}$ zeolite powder was connected to a turbomolecular pump, evacuated, and heated at different temperatures for a certain time. Adsorption and hydrogen isotope exchange experiments were carried out by adding a known aliquot of the corresponding gas at RT to an EPR tube containing $\sim 160 \mathrm{mg}$ of $\mathrm{Pt} / \mathrm{KL}$ zeolite powder. The experiments were monitored via EPR.

In order to determine the absolute coverage of chemisorbed hydrogen, it is necessary to find a reliable procedure to determine the state of zero coverage $\left(\mathrm{Pt}_{13} \mathrm{H}_{0}\right)$ since the EPR signal of the cluster is lost at coverages below $\mathrm{Pt}_{13} \mathrm{H}_{6}$. Fortunately, $\mathrm{H}$ and $\mathrm{D}$ chemisorbed clusters are distinguishable by their EPR spectra, as will be shown in Section 3.5. Therefore, incomplete desorption of $\mathrm{Pt}_{13} \mathrm{H}_{\mathrm{x}}$ and readsorption with $\mathrm{D}$ results in spectra of $\mathrm{Pt}_{13} \mathrm{D}_{\mathrm{x}}$ which are visibly contaminated by $\mathrm{H}$. It was found that complete desorption is reached on evacuation at $573 \mathrm{~K}$ for $3 \mathrm{~h}^{3}$

The spin concentration of Pt/KL zeolite samples was calibrated using a standard sample (ultramarine blue, diluted by $\mathrm{KCl}$ ).

Electron Paramagnetic Resonance. All continuous-wave (c.w.) X-Band EPR spectra were recorded at $4 \mathrm{~K}$ on a Bruker EMX spectrometer with a microwave frequency of about 9.47 $\mathrm{GHz}$ and a power of $1.01 \mathrm{~mW}$. All measurement parameters were kept fixed during a sequence of measurements of one experiment. Pulse X-Band EPR measurements were recorded on a Bruker ElexSys E680X spectrometer with a microwave frequency of about $9.67 \mathrm{GHz}$ at $20 \mathrm{~K}$. The observer position for all HYSCORE spectra was at the magnetic field corresponding to the maximum EPR absorption. The HYSCORE spectra were recorded using a standard four-pulse sequence $\left(\pi / 2-\tau-\pi / 2-t_{1}-\pi-t_{2}-\pi / 2-\tau-\right.$ echo $)$ and an eight-step phase cycle in order to 
remove unwanted echoes. Pulse lengths of $t_{\pi / 2}=t_{\pi}=16 \mathrm{~ns}$, with the $\pi$ pulse at double the MW power to maintain spectral resolution, and a delay time of $\tau=150 \mathrm{~ns}$ were employed. Following common experimental procedures we conducted experiments at variable $\tau$-values (not shown) prior to recording the HYSCORE spectra with a fixed $\tau$-value of $150 \mathrm{ns.} \mathrm{However,} \mathrm{by} \mathrm{setting} \mathrm{up}$ a pulse sequence with an inter-pulse spacing of $\tau=150 \mathrm{~ns}$, there were no 'blind-spots' superimposed on the reported correlation pattern, and simultaneously the obtained modulation depth was at maximum. Therefore, further experiments were done employing a single $\tau$-value, without risking any deterioration of the hyperfine ridges.

The echo is recorded as a function of $t_{1}$ and $t_{2}$, which were incremented independently from each other in steps of $8 \mathrm{~ns}$, starting with initial values of $100 \mathrm{~ns}$. Typically 512 data points were collected in both dimensions. The recorded time-domain data was then processed as follows: the background echo decay in both $t_{1}$ and $t_{2}$ dimension was removed by least-squares fitting to a third-order polynomial and subsequent subtraction. Both dimensions of the data were then zero filled to 1024 data points, convoluted with a Hamming window function and two-dimensional Fourier transformed, giving the frequency-domain HYSCORE spectrum. The spectra presented in this paper are contour plots with linear scaling after magnitude calculation.

EPR spectral simulation was done with the same program as described in the previous work. ${ }^{13}$ High field conditions were assumed so that the effects of the nuclear field on the alignment of the electron spin are negligible, and the electron problem can be treated independently of the nuclei. Treating each nucleus independently allows for more flexibility with near equivalent nuclei. However, it has the disadvantage that the unresolved higher order shifts present for multiple equivalent nuclei lead to anisotropic line shapes are not accounted. For our 
present simulation, this may be responsible in part for the incomplete match of simulated and experimental spectra. For the EPR spectral simulation of the Pt cluster, all possible Pt isotope distributions weighted with the corresponding statistical probabilities were taken into account. ${ }^{13}$ Coaxial hyperfine and $g$ tensors were assumed for the simulation.

SQUID magnetization measurements. Magnetization measurements were performed using a superconducting quantum interference device (SQUID, quantum design MPMXL7). The $M(T)$ measurements were recorded using an applied magnetic field of $2500 \mathrm{G}$. After quantitative hydrogen readsorption the $\mathrm{Pt}_{13}$ cluster samples were transferred into NMR quartz tubes with an outer diameter of $5 \mathrm{~mm}$ under argon atmosphere in the glovebox, and the tubes were sealed with stopcocks for vacuum treatment. This procedure prevents the contact with air. The quartz tubes containing about $60 \mathrm{mg}$ of $5.5 \mathrm{wt} . \% \mathrm{Pt}$ in $\mathrm{KL}(\mathrm{SC})$ zeolite were then fixed in the middle of a plastic straw for SQUID measurements. The susceptibilities were corrected by subtraction of the contribution of the plain zeolite. The effective magnetic moment $\mu_{\mathrm{eff}}$ is obtained in $\mu_{\mathrm{B}}$ from the slope $\mathrm{C}_{\mathrm{m}}\left(\right.$ in $\left.\mathrm{cm}^{3} \mathrm{~K} / \mathrm{mol}\right)$ of the Curie plot via $\mu_{\mathrm{eff}}=2.82 \mathrm{C}_{\mathrm{m}}{ }^{1 / 2}{ }^{15}$

\section{Results and discussion}

Characterization. X-Band EPR spectra in Figure 1 show a multiplet representing a Pt cluster supported on $\mathrm{KL}(\mathrm{CU})$ zeolite. The possibility that the multiplet hyperfine structure originates from the $\mathrm{K}$ nuclei is excluded because $\mathrm{Pt} / \mathrm{LiL}$ and $\mathrm{Pt} / \mathrm{NaL}$ zeolites give the same EPR spectra, while in the absence of Pt no such multiplets are observed. Furthermore, isotopically enriched Pt samples proved unambiguously that the splitting is due to $\mathrm{Pt} .{ }^{13}$ The $g$ value is much larger than $g_{\mathrm{e}}$ of the free electron, which for simple transition metal centers would indicate that the unpaired electron comes from a more than half-filled d shell. ${ }^{16}$ Simulations of the spectra in 
Figure 4 verify that the multiplet is due to a well-defined magic number $\mathrm{Pt}_{13}$ cluster with 12 equivalent Pt atoms on the surface of an icosahedron or cuboctahedron (which are indistinguishable by EPR). The equivalence of the Pt atoms points to Jahn-Teller dynamics. The Pt nucleus in the center of the cluster possesses only a small spin density, which is again understood on the basis of the superatom nature of the cluster which tells that only electrons in $\mathrm{s}$ type superatom orbitals lead to significant spin density at the cluster center. The hyperfine coupling constant of the 12 surface Pt atoms is isotropic and amounts to $A_{\text {iso }} \approx 68.1 \mathrm{G}$. The observed multiplet must be explained as a superposition of spectra of clusters with statistical distribution of all platinum isotopes. With respect to EPR it is only relevant to distinguish between ${ }^{195} \mathrm{Pt}(I=1 / 2$, natural abundance $33.8 \%)$ and all other Pt isotopes with $I=0$. Spectra with an even number of equivalent ${ }^{195} \mathrm{Pt}(I=1 / 2)$ nuclei have a central peak with multiplet lines separated in first order by the hyperfine coupling constant $A^{\mathrm{Pt}}$. For a cluster with an odd number of ${ }^{195} \mathrm{Pt}$ nuclei, the lines are between the peaks of the even spectra. A superposition of these two sets of spectra results in a spectrum consisting of lines split by $A^{\mathrm{Pt}} / 2 .{ }^{13}$ For the as prepared hydrogen reduced $\mathrm{Pt}_{13}$ clusters dispersed in $\mathrm{KL}(\mathrm{CU})$ zeolite EXAFS data reveal a coordination number $\mathrm{CN}(\mathrm{Pt}-\mathrm{Pt})$ of $5.5 \pm 0.6{ }^{3}$ This result agrees very well with the expected value of 5.5 for an ideal cuboctahedron. HRTEM images furthermore confirm that the $\mathrm{Pt}_{13}$ clusters are really small and have a diameter of $1.2(1) \mathrm{nm}^{3}$

Hydrogen desorption and readsorption experiments. Scheme 1 shows a systematic overview of $\mathrm{Pt}_{13}$ cluster states depending on hydrogen coverage at the corresponding equilibrium pressure. It summarizes the results and interpretations reported below, but for better readability it is given before the individual justifications and discussions. 
Scheme 1. Overview over the hydrogen coverage dependent states of the $\mathrm{Pt}_{13}$ cluster

\begin{tabular}{|c|c|}
\hline \multicolumn{2}{|c|}{ State I } \\
\hline \multicolumn{2}{|c|}{ 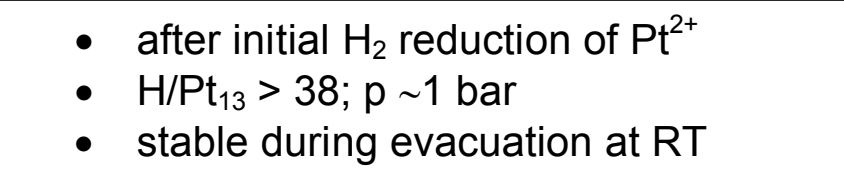 } \\
\hline $\begin{array}{c}\text { gentle } \\
\text { desorption at }\end{array}$ & irreversibl \\
\hline \multicolumn{2}{|c|}{ State II } \\
\hline \multicolumn{2}{|c|}{ - $\mathrm{H} / \mathrm{Pt}_{13}<38 ; \mathrm{p} \sim 500$ mbar } \\
\hline $\begin{array}{l}\text { desorption } \\
\text { up to } 473 \mathrm{~K}\end{array}$ & $\int \begin{array}{c}\text { fully } \\
\text { reversible }\end{array}$ \\
\hline \multicolumn{2}{|c|}{ State III } \\
\hline \multicolumn{2}{|c|}{ - $\mathrm{H} / \mathrm{Pt}_{13}>0 ; \mathrm{p} \sim 0 \mathrm{mbar}$} \\
\hline $\begin{array}{l}\text { fully } \\
\text { reversible }\end{array} / \int_{\text {desc }}^{1}$ & at irreversible \\
\hline State IV & State V \\
\hline $\begin{array}{l}\text { fully desorbed } \\
\text { EPR inactive } \mathrm{Pt}_{13}\end{array}$ & fully desorbed $\mathbf{P t}_{\mathbf{x}}$ \\
\hline
\end{tabular}

Figure 1 shows a hydrogen and deuterium desorption, readsorption, and isotope exchange cycle of the $\mathrm{Pt}_{13}$ cluster states I $(\mathrm{a}, \mathrm{b}), \mathrm{II}(\mathrm{c}, \mathrm{d})$, and III $(\mathrm{e}, \mathrm{f})$ monitored via EPR. It clearly shows that the amount of hydrogen or deuterium coverage has a huge effect on the appearance of the $\mathrm{Pt}_{13}$ cluster multiplet. $\mathrm{H} / \mathrm{D}$ desorption leads to a change in the electronic structure of the $\mathrm{Pt}_{13}$ cluster and to a significant decrease of the $g$ anisotropy (see below). Furthermore, exchange of deuterium for hydrogen leads to slightly narrower lines of the multiplet, resulting in a better resolution. This is due to the fact that the magnetic moment of the proton is a factor 6.5 larger 

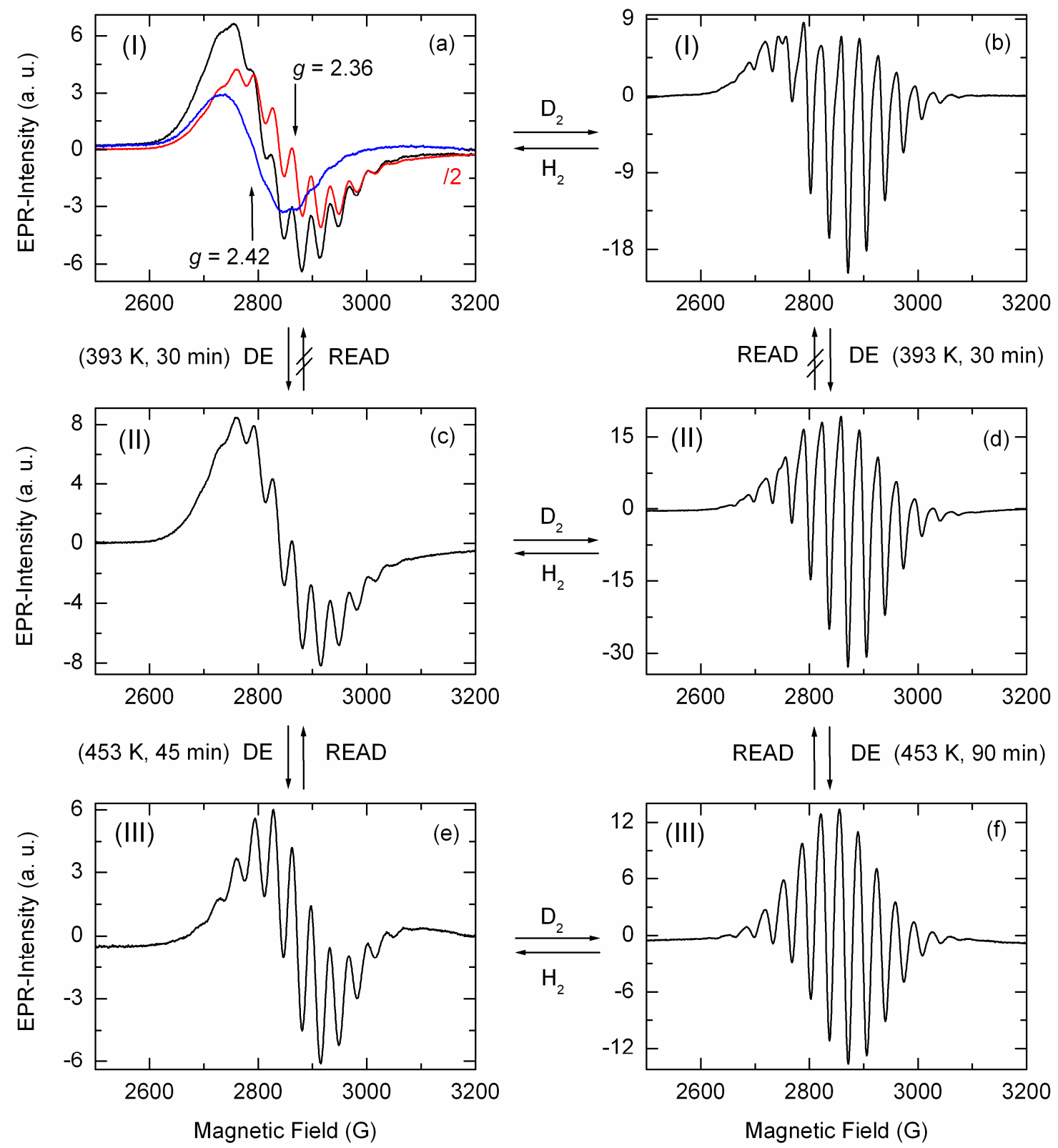

Figure 1. X-Band EPR spectra recorded at $4 \mathrm{~K}$ during the hydrogen and deuterium desorption (DE), readsorption (READ), and isotope exchange cycle of the $\mathrm{Pt}_{13}$ cluster states I (a, b), II (c, d), and III (e, f). The spectra of state I $(\mathrm{a}, \mathrm{b})$ were obtained with a $5.3 \mathrm{wt} . \% \mathrm{Pt} / \mathrm{KL}(\mathrm{CU})$ zeolite after initial hydrogen and deuterium reduction at 1 bar. Gentle desorption of the same samples for $30 \mathrm{~min}$ at $393 \mathrm{~K}$ results in state II (c, d), and following desorption at 453 $\mathrm{K}$ for $\approx 45 \min (\mathrm{e})$ and $\approx 90 \mathrm{~min}$ (f) in state III. The EPR spectrum with $g=2.42$ (a, blue) is the difference spectrum of the spectra (a, black) and (c, $50 \%$ of the intensity) at $g=2.36$ (a, red). 
than that of the deuteron and thus the hyperfine splitting of $\mathrm{H}$ is 6.5 times larger than that for D. ${ }^{13,14}$ The observation implies that the deuteron and proton hyperfine couplings are unresolved but affect the line width, although no strong line width dependence on hydrogen coverage is observed (see below).

It is important to mention that the initial hydrogen or deuterium reduced $\mathrm{Pt}_{13}$ clusters (state I) are unique and stable during isotope exchange and evacuation at RT but are not recovered after desorption even at a gentle temperature of 393 K. Figure 1a shows that the spectrum is a superposition of the spectrum in Figure 1c, with $g=2.36$, and an additional species at $g=2.42$ without any resolved hyperfine structure. By comparison of Figures 1a and 1c it is obvious that it is this last species which is not recoverable after gentle desorption. Similar behavior is found for the deuterium reduced clusters (compare Figures $1 \mathrm{~b}$ and 1d). The spectra of quatitatively readsorbed species do not show the unresolved component (see Figure 3a below). The spectra of the initial reduced $\mathrm{Pt}_{13}$ clusters in $\mathrm{NaY}(\mathrm{CU})$ zeolite did also not show an unresolved component of state I, instead they are analogous to state III, resembling Figure 1e, f. ${ }^{14}$ This clearly indicates that in KL zeolite more hydrogen or deuterium atoms were chemisorbed after the initial reduction of $\mathrm{Pt}_{13}$ clusters than in $\mathrm{NaY}$ zeolite. Accordingly, the zeolite framework has an influence on the properties of the $\mathrm{Pt}_{13}$ clusters. Possible reasons are the different $\mathrm{Si} / \mathrm{Al}$ ratio of $3.0(\mathrm{KL}(\mathrm{CU}))$ and $2.3(\mathrm{NaY}(\mathrm{CU}))$ zeolites as well as the different nature of the charge compensating cations. In agreement with these observations, Ji et al. reported that the hydrogen coverage of $\mathrm{Y}$ zeolite supported $\mathrm{Pt}$ particles with $\mathrm{CN}(\mathrm{Pt}-\mathrm{Pt})$ around 6.5 depends strongly on the support ionicity. ${ }^{17}$ Their experiments demonstrated a clearly decreasing trend of the $\mathrm{H}$ per $\mathrm{Pt}$ value with decreasing electron density on the support oxygen atoms, which is related primarily to the ionic character of the cations in the oxide support. Electron-rich oxygen exist in basic 
supports with large alkaline cations and electron-poor oxygen in acidic supports with protons or other, partly covalent cations. ${ }^{17}$ The alkalinity increases from the charge compensating cation $\mathrm{Na}^{+}$ to $\mathrm{K}^{+}$, which is in line with the argumentation above.

Furthermore, the different structures of the zeolites should also have an influence on the properties of the $\mathrm{Pt}_{13}$ clusters. The framework structure of $\mathrm{NaY}$ zeolite contains the largest void space of any known zeolite. It amounts to about 50 vol.\% compared to about 30 vol.\% for KL zeolite, which possibly affects the resulting crystal field on the $\mathrm{Pt}_{13}$ clusters. ${ }^{18}$ Each $\mathrm{NaY}$ unit cell contains eight supercages with a diameter of $\sim 13 \AA$ and four 12-ring windows of $\sim 7.4 \AA$ diameter. At a 6 wt.\% Pt loading, one $\mathrm{Pt}_{13}$ cluster is accommodated per 25 supercages. In contrast, during hydrogen reduction in the $\mathrm{KL}$ zeolite, the $\mathrm{Pt}_{13}$ clusters are formed in the elliptical cages (diameter $\sim 13 \AA$, width $\sim 4.5 \AA$, and 12-ring windows $\sim 7.1 \AA$ ) of the zeolite channels presented in Figure 2a, b. These hexagonally packed channels (Figure $2 \mathrm{c}$ ) are the main structure element of the L zeolite containing $\mathrm{K}^{+}$in the only exchangeable cation site at $\mathrm{RT} .{ }^{18}$ There is less volume available for the $\mathrm{Pt}_{13}$ cluster in the cages of $\mathrm{KL}$ than in $\mathrm{NaY}$ zeolite. Figure $2 \mathrm{~b}$ presents in correct dimensions a $\mathrm{Pt}_{13}$ cluster (with $\sim 8 \AA$ diameter for the bare cluster) in the elliptical KL zeolite cage. Higher hydrogen coverage should lead to a larger cluster diameter.

Each hydrogen atom requires one electron from the $\mathrm{Pt}_{13}$ cluster orbitals to bind on the cluster surface. Thus, the electron density between the platinum atoms decreases and the Pt-Pt bond length increases from $2.59 \pm 0.02 \AA$ to $2.70 \pm 0.02 \AA$ according to EXAFS results. ${ }^{3}$ This results in a larger diameter of the cluster, and hence the interaction between the Pt cluster and the zeolite framework should be stronger in $\mathrm{KL}$ than in $\mathrm{NaY}$. $\mathrm{The}_{13}$ cluster could therefore be slightly distorted in KL. Nevertheless, the state III spectra are nearly isotropic. 
(a)

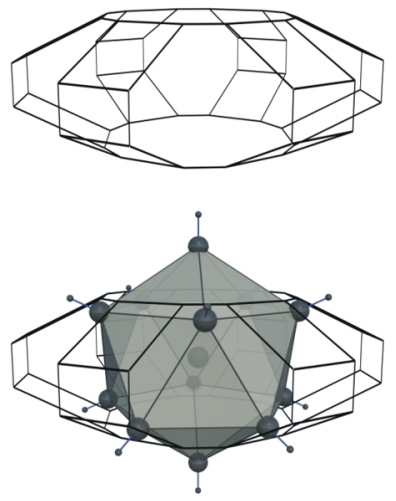

(b) (c)

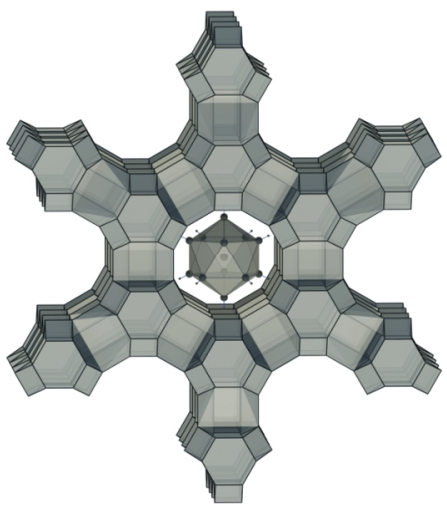

(d)
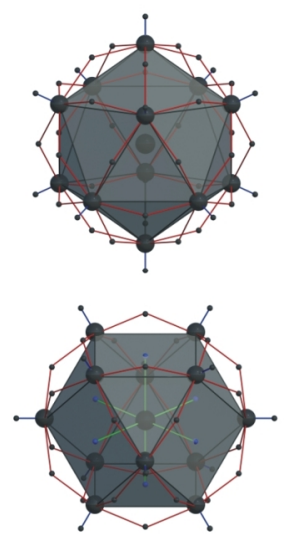

(f) (e)
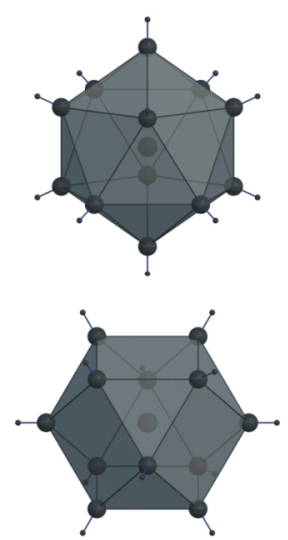

(g)

Figure 2. Elliptical cage of the KL zeolite channel drawn in accurate dimensions (a), and hosting an icosahedral $\mathrm{Pt}_{13} \mathrm{H}_{12}$ cluster (b). Icosahedral $\mathrm{Pt}_{13} \mathrm{H}_{12}$ cluster arranged in one of the hexagonally packed KL zeolite channels (c). Hydrogen chemisorption sites on an icosahedral $\mathrm{Pt}_{13}$ cluster: with 12 occupied corner and 30 edge sites (d), and 12 corner sites (e); and a cuboctahedral cluster with 12 corner, 24 edge, and 6 sites in the four-fold hole (f), and with 12 corner sites (g).

Further desorption of the $\mathrm{Pt}_{13}$ clusters at $453 \mathrm{~K}$, shown in $1 \mathrm{c}$, $\mathrm{d}$ (state II), leads to a decreasing EPR intensity, shown in 1e, $\mathrm{f}$ (state III), but the appearance of the cluster multiplet remains the same. Desorption up to $473 \mathrm{~K}$ results in the disappearance of the $\mathrm{Pt}_{13}$ cluster multiplet (state III). This desorption and readsorption process is fully reversible for desorption temperatures up to approximately $473 \mathrm{~K}$, leading again to $1 \mathrm{c}, \mathrm{d}$ (state II) with the same $\mathrm{Pt}_{13}$ cluster EPR intensities (see also figure 3a below). Cross-readsorption of formerly hydrogen covered $\mathrm{Pt}_{13}$ clusters with deuterium gas and vice versa after desorption at $\sim 473 \mathrm{~K}$ show an EPR multiplet resulting from a superposition of hydrogen and deuterium covered $\mathrm{Pt}_{13}$ clusters (not shown). Obviously, a desorption temperature of $473 \mathrm{~K}$ is not enough for a complete desorption of the chemisorbed hydrogen, and a fraction of the H/D atoms remain on the cluster surface while 
the EPR activity is already lost. However, at a desorption temperature of $573 \mathrm{~K}$ which leads to completely desorbed $\mathrm{Pt}_{13}$ clusters (state IV) and to an irreversible restructured, and fully desorbed platinum species $\mathrm{Pt}_{\mathrm{X}}$ (state $\mathrm{V}$ ), the cross-readsorption experiment reveals full desorption, as shown in Figure 3. Although we do not have more specific information about the difference between state IV and state $\mathrm{V}$ this desorption condition therefore provides a reliable starting point for quantitative hydrogen readsorption experiments. Further details about state $\mathrm{V}$ are found in the Section on Oxygen Adsorption.

The splitting pattern of the $\mathrm{Pt}_{13}$ cluster multiplet disappeared completely after full desorption, and a broad unresolved signal appeared at $g=2.18$ (state $\mathrm{V}$ ), resulting from another platinum species, as reported also in previous work. ${ }^{3}$ Furthermore, it is important to assess that after the complete H/D desorption treatment at $573 \mathrm{~K}$ for $3 \mathrm{~h}$ the EPR intensity of the gently predesorbed $\mathrm{Pt}_{13}$ clusters as in Figure 1e, $\mathrm{f}$ (state III) is not fully recovered, but the main features of the $\mathrm{Pt}_{13}$ cluster multiplet remain the same as demonstrated in Figure 3 and elsewhere. ${ }^{3}$ On hydrogen desorption at $573 \mathrm{~K}$, structural modifications are observed, as the total $\mathrm{Pt}-\mathrm{Pt}$ coordination number descreases, ${ }^{3}$ which reminds of the predicted coverage-dependent reconstruction for $\gamma-\mathrm{Al}_{2} \mathrm{O}_{3}$ supported $\mathrm{Pt}_{13}$ clusters reported by Mager-Maury et al., ${ }^{4}$ or perhaps to some extent a disintegration. 

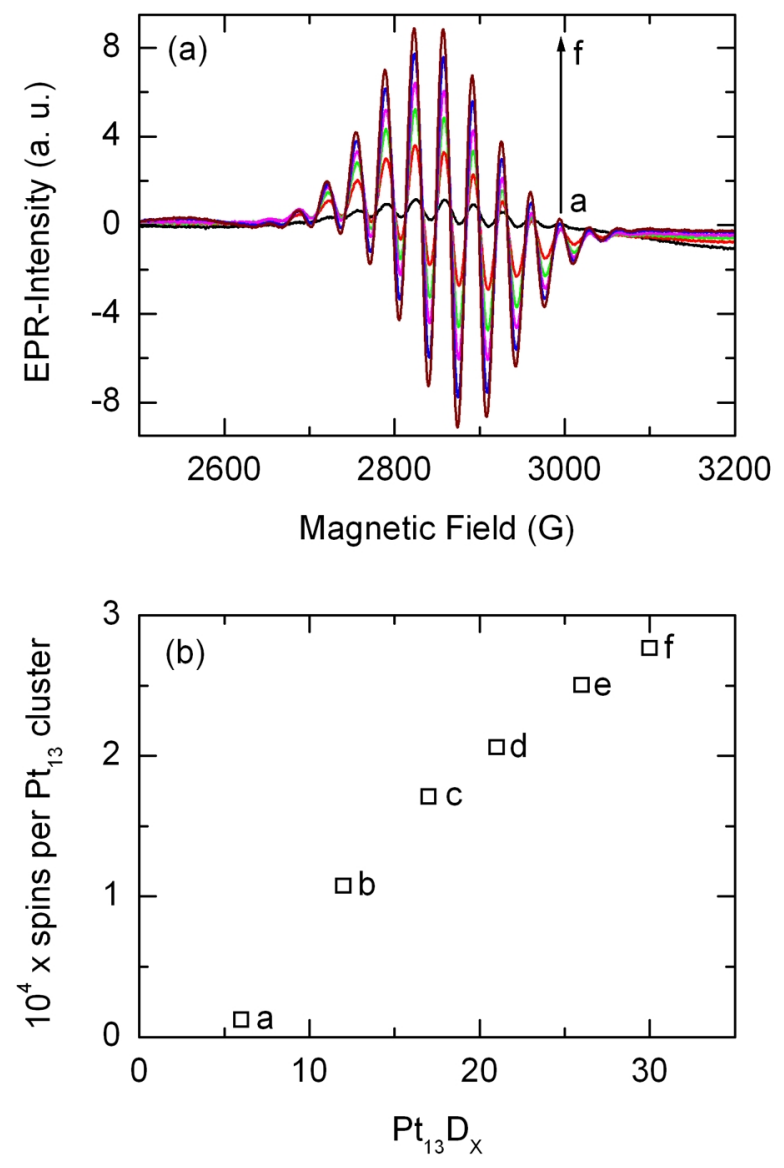

Figure 3. (a) Increasing EPR intensity during gradual quantitative deuterium cross-readsorption of a formerly hydrogen covered $\mathrm{Pt}_{13}$ cluster dispersed in $\mathrm{KL}(\mathrm{CU})$ zeolite after complete desorption at $573 \mathrm{~K}$ for $3 \mathrm{~h}$. The arrow indicates the direction of increasing deuterium coverage. (b) Absolute spin concentration as a function of deuterium coverage: a $\left(0\right.$ mbar, $\left.\mathrm{Pt}_{13} \mathrm{D}_{6}\right), \mathrm{b}\left(0.02\right.$ mbar, $\left.\mathrm{Pt}_{13} \mathrm{D}_{12}\right)$, c (0.47 mbar, $\left.\mathrm{Pt}_{13} \mathrm{D}_{17}\right), \mathrm{d}\left(2.4\right.$ mbar, $\left.\mathrm{Pt}_{13} \mathrm{D}_{21}\right)$, e (34 mbar, $\mathrm{Pt}_{13} \mathrm{D}_{26}$ ), and $\mathrm{f}\left(70\right.$ mbar, $\mathrm{Pt}_{13} \mathrm{D}_{30}$ ).

Inspection of Figure $3 \mathrm{a}$ shows that between the compositions $\mathrm{Pt}_{13} \mathrm{D}_{6}$ and $\mathrm{Pt}_{13} \mathrm{D}_{30}$ the EPR spectra change only by an amplitude scaling factor. It seems surprising that the Pt hyperfine coupling that reflects the electron spin density at the Pt nucleus is not affected by the coverage, and that the Pt atoms do not become inequivalent. The latter point could be explained by dynamics, but at $4 \mathrm{~K}$ this is a risky interpretation. For the independent hyperfine coupling we suggest that at a given nominal coverage we actually have a broad equilibrium distribution of 
coverages, but only one or a few of these coverages near the maximum value has a spin $S=1 / 2$ and is EPR active (see below). By adding more deuterium the average coverage shifts to higher values, which increases also the fraction of EPR active clusters without changing their composition. Below the average composition of $\mathrm{Pt}_{13} \mathrm{D}_{6}$ no EPR active clusters remain detectable. Above it, Figure $3 b$ reveals a nearly linear increase of the EPR signal intensity with deuterium coverage. This behavior is essential for the interpretation of the desorption experiments. The maximum spin concentration that is reached at a deuterium pressure of 70 mbar, corresponding to a composition of $\mathrm{Pt}_{13} \mathrm{D}_{30}$, amounts to ca. $3 \times 10^{-4}$ spins per $\mathrm{Pt}_{13}$ cluster.

We close this section with a discussion about the unique state I of the initial hydrogen and deuterium reduced $\mathrm{Pt}_{13}$ clusters shown in Figures $1 \mathrm{a}$ and $\mathrm{b}$. As mentioned above the initial reduced state I which is stable during isotope exchange and evacuation at RT is a superposition of two species. The structureless spectrum at $g=2.42$ is not recovered on readsorption from state II. It is possible that this behavior relates to a structural transition similar to the one reported by Chen et al. in a theoretical study of hydrogen chemisorption on $\mathrm{Pt}_{13}$ clusters where they described a transition from an icosahedral to a fcc-like structure when more than 8 hydrogen atoms are chemisorbed, ${ }^{5}$ which is compatible with EXAFS results ${ }^{3}$. The saturation point was predicted to be reached at 44 chemisorbed hydrogen atoms resulting in a value of around $3.4 \mathrm{H} / \mathrm{Pt}$. In total, 36 hydrogen atoms can chemisorb at the edge and corner sites of the fcc $\mathrm{Pt}_{13}$ cluster surface. Additional $\mathrm{H}$ atoms can penetrate through the six four-fold holes into the cluster and interact with the core Pt atom. ${ }^{5}$ Furthermore, recent DFT calculations by Mager-Maury et al. for $\gamma-\mathrm{Al}_{2} \mathrm{O}_{3}$ supported bare $\mathrm{Pt}_{13}$ clusters predict a reconstruction from a biplanar morphology on the support to a cuboctahedral structure at a coverage near $18 \mathrm{H}$ per cluster, and a maximum coverage of 3 
$\mathrm{H} / \mathrm{Pt}^{4}{ }^{4}$ The different chemisorption sites for icosahedral and cuboctahedral $\mathrm{Pt}_{13}$ clusters are presented in Figure 2d-g.

Quantitative hydrogen readsorption experiments after complete desorption at $573 \mathrm{~K}$ for 3 $\mathrm{h}$ were carried out on $\mathrm{KL}(\mathrm{SC})$ zeolite supported $\mathrm{Pt}_{13}$ clusters. For a final $\mathrm{H}_{2}$ pressure of $540 \mathrm{mbar}$ they lead to a value of $38 \pm 2$ chemisorbed hydrogen atoms on the $\mathrm{Pt}_{13}$ cluster surface $(\sim 2.9 \pm 0.2$ $\mathrm{H} / \mathrm{Pt}$ ) as reported elsewhere. ${ }^{3}$ As demonstrated in Scheme 1 and Figure 1 it is not possible by hydrogen or deuterium readsorption to achieve the initial reduced state I and a H/D coverage $>38$ $\mathrm{H}$ atoms. Possibly, an irreversible phase transition near the coverage of $38 \pm 2 \mathrm{H} / \mathrm{D}$ atoms from a cuboctahedral state I to an icosahedral state II takes place during gentle desorption of the initial reduced state I. This means that the initial reduced state I in the form of a H/D covered cuboctahedral $\mathrm{Pt}_{13}$ cluster only exists after the natural cluster formation during hydrogen and deuterium reduction and finally disappears after $\mathrm{H} / \mathrm{D}$ desorption even at gentle temperatures.

Simulation of EPR spectra. The EPR spectra shown in Figure $4 a-c$ demonstrate the correlation between the amount of deuterium coverage increasing from (a) to (c) on the $\mathrm{Pt}_{13}$ cluster surface and the $g$ anisotropy, which provides an insight into the electronic structure of the cluster. Experimental EPR spectra are in black and the simulated spectra are red. All spectra in Figure 4 are simulated for 12 equivalent Pt nuclei. Since the hydrogen couplings are not resolved in the experiment they were omitted in the simulations and are absorbed by the intrinsic line widths. Simulations were performed with variable $g$ anisotropy only, since the hyperfine tensor $A$ was found to remain isotropic. Due to $g$ anisotropy, the extension of the lines above and below the base line are not equal, as can be seen clearly in Figure 4 by inspection of the EPR intensity values on the axis. Furthermore, $g$ anisotropy contributes equally to broadening of all hyperfine components, and the effect scales with the magnetic field. $A$ anisotropy would affect the outer 
lines more strongly. Figure $4 \mathrm{a}$ and $1 \mathrm{f}$ show deuterium covered $\mathrm{Pt}_{13}$ clusters after desorption for $\sim 90 \mathrm{~min}$ at $453 \mathrm{~K}$ (state III) as examples for partial deuterium coverage. This state is also reversibly accessible after complete desorption and readsorption treatment. Figure $4 \mathrm{~b}$ and $1 \mathrm{~d}$ (state II) represents intermediate deuterium coverage. This state is accessible both after gentle desorption and after full desorption followed by readsorption under rising pressure up to $\sim 600$ mbar. Finally, Figure $4 \mathrm{c}$ is used as an example for initially deuterium reduced and most highly covered $\mathrm{Pt}_{13}$ clusters (state $\mathrm{I}$ ). The simulations $4 \mathrm{a}$ and $\mathrm{b}$ fit really well, which is a sensitive confirmation that the assumed cluster structure with 12 equivalent $\mathrm{Pt}$ atoms is correct. $4 \mathrm{c}$ is less accurate since it does not account for the superposition of a second species with a broad, unresolved, single-line spectrum (compare Figure 1a). Nevertheless Figure 4 clearly shows the correlation between the coverage and the $g$ anisotropy. Additionally, the spectrum of the $\mathrm{Pt}_{13} \mathrm{H}_{\mathrm{X}}$ clusters in state III shown in Figure 1e was simulated.
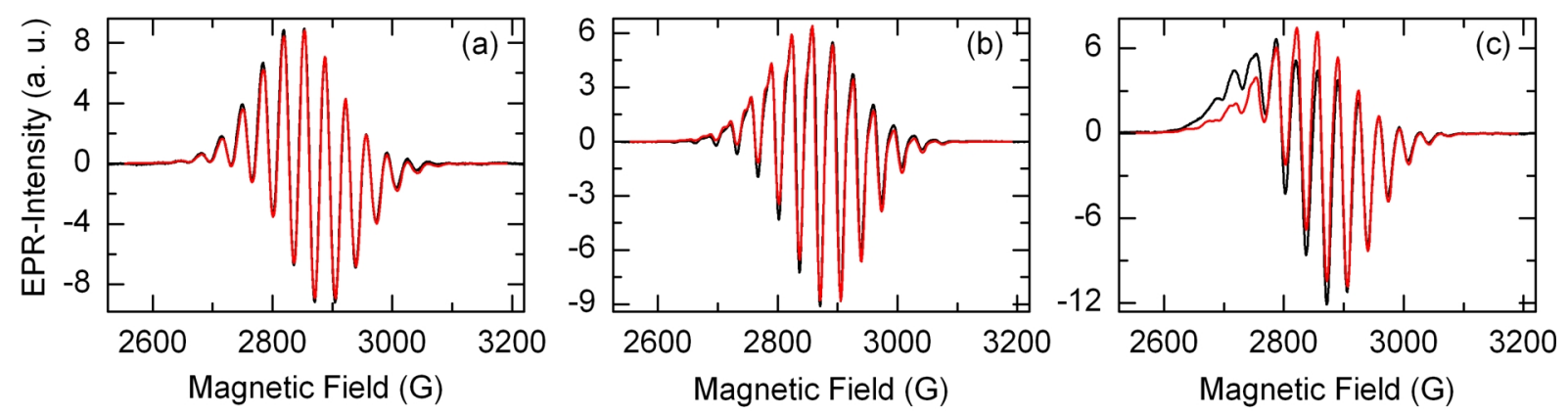

Figure 4. Development of the EPR spectra with the extent of deuterium coverage increasing from (a) to (c) on the $\mathrm{Pt}_{13}$ cluster surface and the $g$ anisotropy; experiment (black), simulation (red). (a) Low, (b) intermediate, and (c) complete deuterium coverage.

The fit parameters are collected in Table 1. The isotropic $g$ value increases slightly with increasing D coverage, and for $\mathrm{H}$ coverage in the same state it is significantly higher for reasons which are not well understood at this point. The $g$ anisotropy is a strong function of $\mathrm{D}$ coverage, 
as commented above from qualitative inspection of Figure 4. This is obviously a consequence of the occupation of different cluster molecular orbitals by the unpaired electron. The Pt hyperfine coupling is independent of D coverage, but for $\mathrm{H}$ coverage it is slightly lower, perhaps due to the fact that for an anharmonic potential the $\mathrm{Pt}-\mathrm{H}$ bond is slightly longer than $\mathrm{Pt}-\mathrm{D}$ in the vibrational average. The value of the intrinsic line width is almost double for the $\mathrm{H}$ covered cluster because of the unresolved hydrogen coupling which is larger for $\mathrm{H}$ than for $\mathrm{D}$ due to the larger nuclear magnetic moment. One might have expected a significant coverage dependence of the line width. The fact that this is not the case may be related to the above proposal that only a small range of hydrogen coverages lead to EPR active clusters.

Table 1. EPR Parameters from the simulation of experimental spectra with 12 equivalent Pt nuclei

\begin{tabular}{|c|c|c|c|c|c|c|c|c|c|c|}
\hline \multirow{2}{*}{ Figure } & \multirow{2}{*}{ State } & \multicolumn{4}{|c|}{$g$} & \multicolumn{4}{|c|}{$A_{\mathrm{Pt}}(\mathrm{G})$} & \multirow{2}{*}{$\operatorname{lw}(\mathrm{G})^{*}$} \\
\hline & & $g_{\perp}$ & $g_{\|}$ & $g_{\|}-g_{\perp}$ & $g_{\text {iso }}$ & $A_{\perp}$ & $A_{\|}$ & $A_{\|}-A_{\perp}$ & $A_{\text {iso }}$ & \\
\hline $4 a, 1 f$ & III (D) & 2.3615 & 2.3795 & 0.0180 & 2.3675 & 68.1 & 68.1 & 0 & 68.1 & 5.7 \\
\hline $4 \mathrm{~b}, 1 \mathrm{~d}$ & II (D) & 2.3611 & 2.3836 & 0.0225 & 2.3686 & 68.1 & 68.1 & 0 & 68.1 & 4.2 \\
\hline $4 c, 1 b$ & $\mathrm{I}(\mathrm{D})^{\Uparrow}$ & 2.3612 & 2.4131 & 0.0519 & 2.3785 & 68.1 & 68.1 & 0 & 68.1 & 5.5 \\
\hline $1 \mathrm{e}^{3}$ & III $(\mathrm{H})$ & 2.3528 & 2.3729 & 0.0201 & 2.3595 & 67.1 & 68.1 & 1 & 67.4 & 9.0 \\
\hline
\end{tabular}

${ }^{*}$ intrinsic (single crystal) line width

"resolved multiplet

HYSCORE experiments. Two models are conceivable for desorption of H/D covered $\mathrm{Pt}_{13}$ clusters. The first model includes that after the initial hydrogen or deuterium reduction (state I) weakly and strongly bonded H/D atoms are already present at energetically different chemisorption sites on the $\mathrm{Pt}_{13}$ cluster surface as shown in Figure $2 \mathrm{~d}-\mathrm{g}$. With increasing desorption first the weakly bonded and subsequently the more strongly bonded H/D atoms are 
desorbed. The second model implies that after the initial reduction (state I) all H/D atoms are bonded equally, and that after desorption of part of the hydrogen or deuterium the remaining H/D atoms are more strongly bonded. This can be due to a change in the electronic properties of the $\mathrm{Pt}_{13}$ clusters. $^{19}$

A sensitive method to probe hydrogen chemisorption at the surface of the $\mathrm{Pt}_{13}$ clusters is provided by HYSCORE. In this experiment, a mixing $\pi$-pulse correlates the nuclear transition frequencies $v_{\alpha}$ and $v_{\beta}$ of the two electron spin manifolds and leads to cross peaks at $\left(v_{\alpha}, v_{\beta}\right)$ and $\left(v_{\beta}, v_{\alpha}\right) \cdot{ }^{20}$ Furthermore HYSCORE spectra can clearly differentiate between a distribution of isotropic couplings and a broadening due to dipolar couplings. This is because in HYSCORE spectra the dipolar hyperfine interactions are not only determined by the length of the cross-peak ridges, but also by the shift of the cross peaks by $\Delta v_{S}$ from the anti-diagonal of the 2-D plot (see Figure 5). ${ }^{21}$ For weak hyperfine interactions and in the limit of the point-dipole approximation at distance $r$, the dipole interaction $T$ is calculated from $\Delta v_{S}$ by

$$
T=\frac{4}{3}\left(2 v_{I} \Delta v_{S}\right)^{\frac{1}{2}}=\frac{\mu_{0}}{4 \pi} \frac{g_{e} g_{N} \beta_{e} \beta_{N}}{h} \cdot\left\langle\frac{1}{r^{3}}\right\rangle
$$

A HYSCORE spectrum of initial hydrogen reduced $\mathrm{Pt}_{13}$ clusters (state I) dispersed in $\mathrm{KL}(\mathrm{CU})$ zeolite recorded at a field of $2900 \mathrm{G}$ and a temperature of $20 \mathrm{~K}$ is displayed in Figure $5 \mathrm{a}$. If proton hyperfine couplings are smaller than the proton Zeeman frequency one normally calls them "weakly coupled" protons. In the following we have to distinguish between two types of weakly coupled protons. The two ridges along the anti-diagonal in Figure 5a are separated by about $A_{\text {iso }}{ }^{\mathrm{H}}=12.4 \mathrm{MHz}\left(A_{\perp}{ }^{\mathrm{H}}=10.3 \mathrm{MHz}, A_{\|}{ }^{\mathrm{H}}=16.6 \mathrm{MHz}\right)$ and represent the more strongly coupled of these protons. Data analysis reveals that the ridges have a maximum frequency shift of 
$\Delta v_{S}=0.25 \pm 0.10 \mathrm{MHz}$, corresponding to a dipolar hyperfine coupling of $T=3.3 \pm 0.1 \mathrm{MHz}$. Based on the point-dipole approximation (equation 3) we obtain a point-dipole distance of the hydrogen nuclei to the unpaired electron of about $3.05 \pm 0.20 \AA$. Figure $5 \mathrm{~b}$ shows a HYSCORE spectrum of quantitatively hydrogen readsorbed $\mathrm{Pt}_{13} \mathrm{H}_{38}$ clusters (state II) supported on $\mathrm{KL}(\mathrm{SC}$ ) zeolite after complete desorption at $573 \mathrm{~K}$ for $3 \mathrm{~h}$. The spectrum is recorded at a field of $2970 \mathrm{G}$ and a temperature of $20 \mathrm{~K}$. The diagonal peak at $\left(v_{\mathrm{H}}, v_{\mathrm{H}}\right)$ with the ridge at $v_{\mathrm{H}}=12.6 \mathrm{MHz}$ is due to protons which interact with the unpaired electron only by dipolar coupling (perhaps nearby matrix protons). The remaining four ridges along the anti-diagonal represent two different kinds of hyperfine coupled protons. The external ridges are separated by about $A_{\text {iso }}{ }^{\mathrm{H} 1}=13.3 \mathrm{MHz}\left(A_{\perp}{ }^{\mathrm{H} 1}=\right.$ 11.7 MHz, $A_{\|}^{\mathrm{H} 1}=16.6 \mathrm{MHz}$ ), which is similar but still significantly more than in Figure 5a. They have a maximum frequency shift of $\Delta v_{S}=0.25 \pm 0.10 \mathrm{MHz}$, corresponding to a dipolar hyperfine coupling of $T=3.3 \pm 0.1 \mathrm{MHz}$, from which we derive a point-dipole distance of the hydrogen nuclei to the unpaired electron of about $3.05 \pm 0.20 \AA$. The inner ridges are separated by about $A_{\text {iso }}{ }^{\mathrm{H} 2}=6.6 \mathrm{MHz}\left(A_{\perp}^{\mathrm{H} 2}=4.7 \mathrm{MHz}, A_{\|}^{\mathrm{H} 2}=10.3 \mathrm{MHz}\right)$ and have a maximum frequency shift of $\Delta v_{S}$ $=0.15 \pm 0.1 \mathrm{MHz}$, corresponding to a dipolar hyperfine coupling of $T=2.6 \pm 0.1 \mathrm{MHz}$. Based on the point-dipole approximation we obtain a distance of the hydrogen nuclei to the delocalized unpaired electron of about $3.3 \pm 0.2 \AA$. 

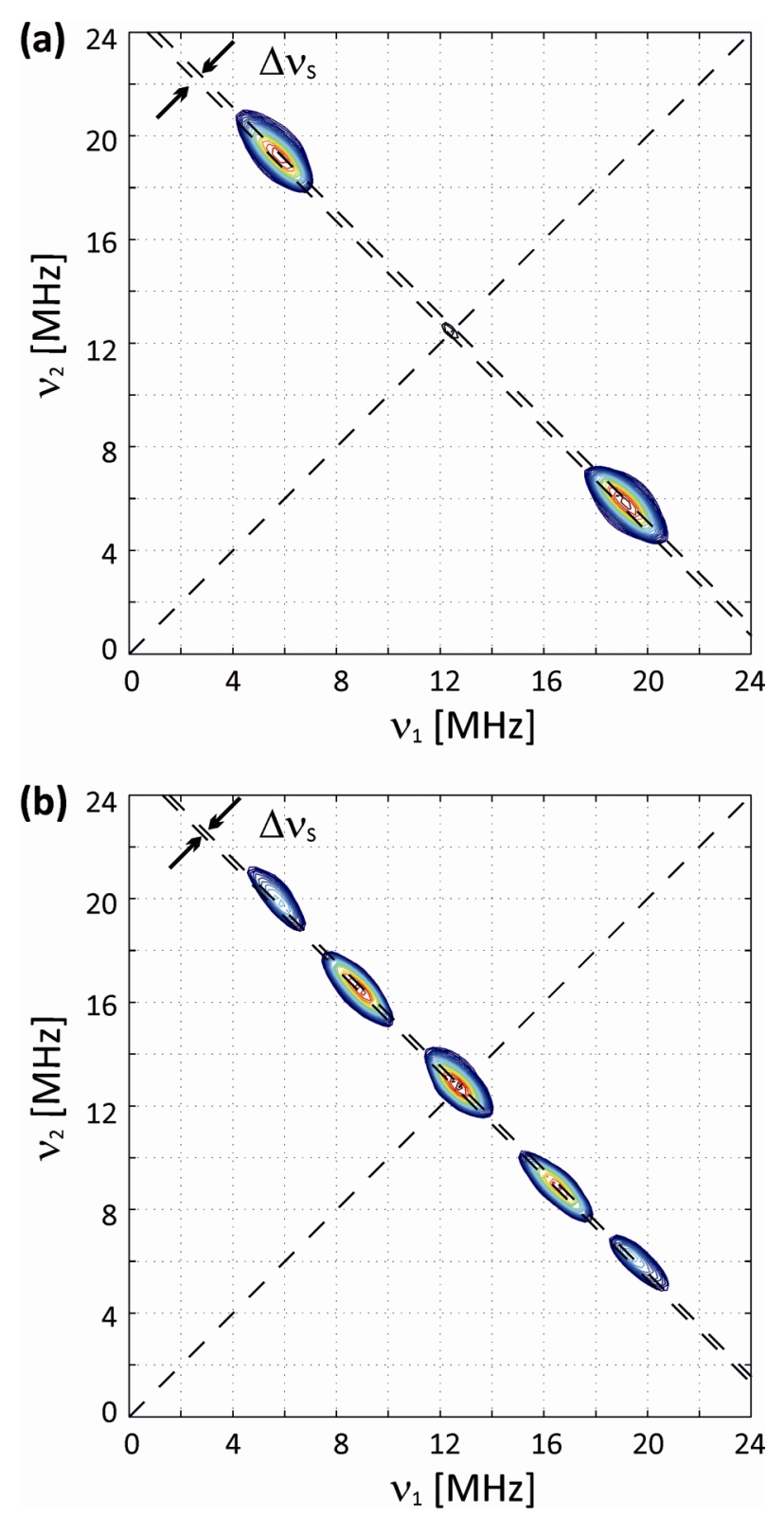

Figure 5. (a) HYSCORE spectrum of initial hydrogen reduced $\mathrm{Pt}_{13}$ clusters (state I) dispersed in KL(CU) zeolite recorded at a field of $2900 \mathrm{G}$ and a temperature of $20 \mathrm{~K}$. The two patterns along the anti-diagonal represent the more strongly coupled protons on the $\mathrm{Pt}_{13}$ cluster surface. (b) HYSCORE spectrum of quantitatively hydrogen readsorbed $\mathrm{Pt}_{13} \mathrm{H}_{38}$ clusters (state II) supported on $\mathrm{KL}(\mathrm{SC})$ zeolite after complete desorption at $573 \mathrm{~K}$ for $3 \mathrm{~h}$ recorded at a field of $2970 \mathrm{G}$ and a temperature of $20 \mathrm{~K}$. The diagonal peak at $v_{\mathrm{H}}=12.6 \mathrm{MHz}$ is due to protons without any isotropic hyperfine coupling, the other four ridges along the anti-diagonal represent two different 
kinds of weakly coupled protons. Numerical simulations of the two sets of ridges are given in the Electronic Supplementary Information.

$\mathrm{Pt}_{13}$ clusters dispersed in $\mathrm{KL}(\mathrm{CU})$ zeolite after gentle desorption at $393 \mathrm{~K}$ for 30 min (state II) recorded with the same parameters as used in Figure 5a show an identical HYSCORE spectrum (not shown) as presented in Figure 5a for state I. While all hydrogen atoms in Figure 5a are equivalent it was shown in Figure 1a that the spectrum of state I represents a superposition of two EPR active species. A possible explanation is that the species with the unresolved spectrum at $g=2.42$ has inequivalent $\mathrm{Pt}$ atoms and therefore also a distribution of proton couplings so that the corresponding HYSCORE signals become weaker. Indeed, if the threshold for display is lowered in Figure 5a two further magnetically inequivalent protons with smaller hyperfine coupling appear above noise level, although with clearly smaller intensity than the two main ridges (not shown). Also the $\mathrm{Pt}_{13}$ clusters supported on $\mathrm{NaY}(\mathrm{CU})$ zeolite showed only one type of hydrogen nuclei after initial hydrogen reduction. ${ }^{14}$

HYSCORE measurements as well as readsorption experiments indicate that the treatment for a full desorption $(573 \mathrm{~K}, 3 \mathrm{~h})$ of the $\mathrm{Pt}_{13}$ clusters has an influence on their properties, leading to a decreasing intensity of $S=1 / 2 \mathrm{Pt}_{13}$ clusters after readsorption. EXAFS data reveal that a fraction of the $\mathrm{Pt}_{13}$ clusters possibly underwent restructuring, resulting in a decreasing $\mathrm{Pt}-\mathrm{Pt}$ coordination number or becoming EPR invisible as reported also in previous work. ${ }^{3}$ Hydrogen readsorbed $\mathrm{Pt}_{13}$ clusters supported in $\mathrm{KL}(\mathrm{SC})$ zeolite show two different kinds of chemisorbed hydrogen nuclei and clearly energetically different adsorption sites on the cluster surface compared to the initial reduced clusters (state I). It is furthermore important to mention that HYSCORE spectra recorded at a field of $2970 \mathrm{G}$ and a temperature of $20 \mathrm{~K}$ for readsorbed 
hydrogen $\mathrm{Pt}_{13} \mathrm{H}_{12}$ (not shown), $\mathrm{Pt}_{13} \mathrm{H}_{23}$ (not shown), and $\mathrm{Pt}_{13} \mathrm{H}_{38}$ (Figure 5b) clusters show identical peak positions.

Figure 6 presents a projection of the ${ }^{1} \mathrm{H}$ cross peaks in the two-dimensional HYSCORE data onto one of the nuclear frequency axes in order to compare relative variations in intensity which are related to the relative amounts of the different proton chemisorption sites. The adsorption sites of the more strongly coupled hydrogen nuclei (the external ridges) are already saturated after quantitative readsorption of twelve hydrogen atoms. Further hydrogen readsorption to $\mathrm{Pt}_{13} \mathrm{H}_{23}$ leads to an increase of the inner ridges (more weakly coupled protons) and to further occupation of this chemisorption site. Raising the pressure further to reach $\mathrm{Pt}_{13} \mathrm{H}_{38}$ results in a further increasing HYSCORE intensity of the inner ridges on the anti-diagonal as well as of the dipolar coupled protons on the diagonal. From the relative intensities it is clear that more adsorption sites are available for the more weakly coupled hydrogens corresponding to the inner ridges on the anti-diagonal.

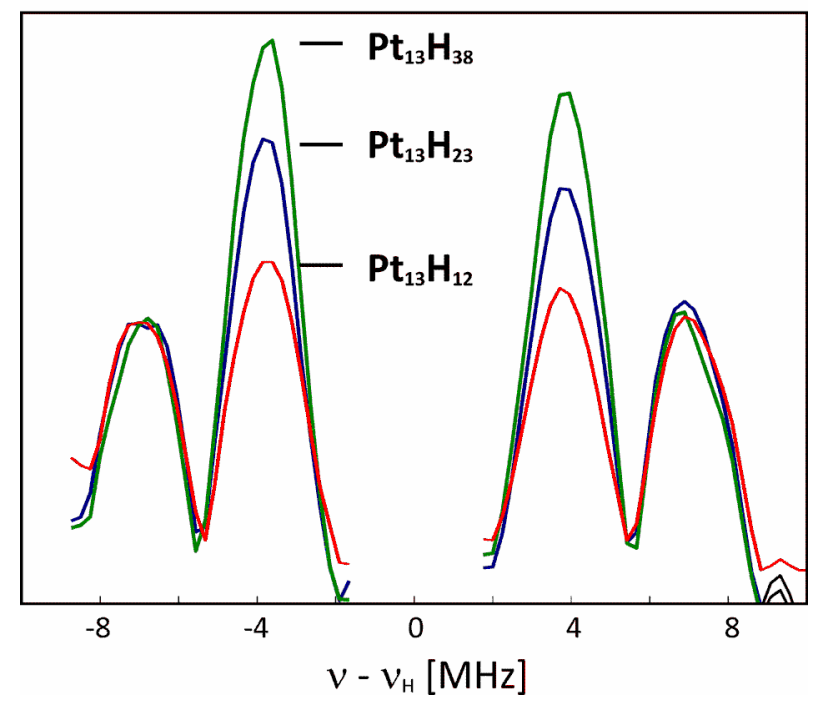

Figure 6. Projection of the ${ }^{1} \mathrm{H}$-correlation peaks of the two-dimensional HYSCORE spectra onto one nuclear frequency axis for the samples $\mathrm{Pt}_{13} \mathrm{H}_{12}$ (red), $\mathrm{Pt}_{13} \mathrm{H}_{23}$ (blue), and $\mathrm{Pt}_{13} \mathrm{H}_{38}$ (green). For comparison, the absolute 
intensity of the projections is scaled to the intensity of the correlation peaks with the strongest hyperfine coupling. The original spectra which include two artifacts are displayed in the Electronic Supplementary Information.

A quantitative interpretation of the intensities in Figure 6 is difficult since the spectra are scaled and the intensities often depend on the size of the hyperfine coupling.

Why then is the $6.6 \mathrm{MHz}$ signal only observed after complete $\mathrm{H}$ desorption and readsorption but not after the initial reduction and partial desorption? In fact the larger coupling is significantly different before complete desorption $(12.4 \mathrm{MHz})$ than after readsorption (13.3 $\mathrm{MHz}$ ), suggesting that the two species are not identical and that before desorption the clusters may be icosahedral, with 30 equivalent edge sites. Note that DFT calculations predicted a coverage-dependent structure change from cuboctahedral to icosahedral ${ }^{5}$ which also depends on the nature of the support. ${ }^{4}$ The EXAFS derived structural change is more extensive, but it represents an average over all Pt species while only ca. $1 \%$ is EPR active.

Desorption Energy. Since desorption is faster at higher temperatures we can determine its activation energy, and since the reverse process is not significantly activated the activation energy of desorption equals the desorption energy. Desorption experiments with the initial hydrogen and deuterium reduced $\mathrm{Pt}_{13}$ clusters (state I) at gentle temperatures between approximately $393 \mathrm{~K}$ and $423 \mathrm{~K}$ for $\sim 30 \mathrm{~min}$ at first always result in a rising EPR intensity of the $\mathrm{Pt}_{13}$ cluster multiplet. Furthermore, deuteration of EPR active hydrogen covered $\mathrm{Pt}_{13}$ clusters always results in a higher EPR intensity by a factor of $2-3$, which means that the deuterated clusters are thermodynamically favored over their hydrogen analogs. This applies for initial H/D reduction (state I) as well as for H/D isotope exchange (see Figure 1). Obviously, the deuterated species have a different the zero-point vibrational energy. Thus, the EPR visible $\mathrm{Pt}_{13}$ clusters are in equilibrium with a reservoir of similar but EPR invisible clusters (e.g. diamagnetic or high-spin 
clusters). Should they be EPR active only in a narrow range below the maximum coverage (see discussion to Figure 3), then partial desorption of fully covered clusters would shift the distribution to the EPR active range, leading to the observed signal increase. Possible reasons for the coexistence of several electronic and magnetic states of similar clusters in the same support are different environments, e.g. because of the inhomogeneous distribution of $\mathrm{Al}$ sites, besides the distribution in $\mathrm{H} / \mathrm{D}$ coverages.

The following desorption experiments for the determination of the $\mathrm{D}_{2}$ desorption energy were carried out with a deuterium covered sample at 453,463 , and $473 \mathrm{~K}$. First, the sample was desorbed at $423 \mathrm{~K}$ for $60 \mathrm{~min}$ to eliminate the initially rising EPR intensity resulting from the $\mathrm{Pt}_{13}$ cluster equilibrium as described above. This procedure ensures a continuous decrease of the EPR intensity on incremental desorption. The results are displayed in Figure 7a. After each completed desorption experiment at a given temperature the EPR tube was filled with $\sim 600 \mathrm{mbar}_{2}$ gas and left overnight. The desorption and readsorption process between state II and III is completely reversible up to $\sim 473 \mathrm{~K}$, as demonstrated by equal intensities at the beginning of each desorption experiment. As expected for an activated process, the EPR intensity decreases faster at the higher desorption temperature. Hydrogen or deuterium desorption from the cluster surface involve the recombination of two atoms, which could give rise to second order kinetics. However, the experimental data fitted well with exponential decay curves with a small positive constant term of $<10 \%$ of the initial intensity. This is compatible with first order kinetics, implying that the ratedetermining step is desorption rather than recombination.

As mentioned above, full desorption of the cluster surface requires a desorption treatment of $573 \mathrm{~K}$ for $\sim 3 \mathrm{~h}$. The desorption time constants derived from Figure $7 \mathrm{a}$ are given in an Arrhenius plot in Figure 7b, from which a value of $2.1 \pm 0.2 \mathrm{eV}$ for the $\mathrm{D}_{2}$ desorption energy from 
the $\mathrm{Pt}_{13}$ cluster dispersed in $\mathrm{KL}(\mathrm{CU})$ zeolite is calculated. A similar desorption experiment of deuterium covered $\mathrm{Pt}_{13}$ clusters supported in $\mathrm{NaY}(\mathrm{CU})$ zeolite resulted in a value of $1.36 \mathrm{eV}$ for the $\mathrm{D}_{2}$ desorption energy. ${ }^{14}$ Comparison of these two values reflect a significant support effect which becomes obvious already from the fact that desorption from clusters with an identical EPR spectrum requires a $\sim 55 \mathrm{~K}$ higher temperature in $\mathrm{KL}$ than in $\mathrm{NaY}$ zeolite. After the higher chemisorption capacity and presence of a phase transition mentioned above, this is the third large difference in the properties of $\mathrm{Pt}_{13}$ clusters supported in $\mathrm{KL}(\mathrm{CU})$ vs. $\mathrm{NaY}(\mathrm{CU})$ zeolite. The
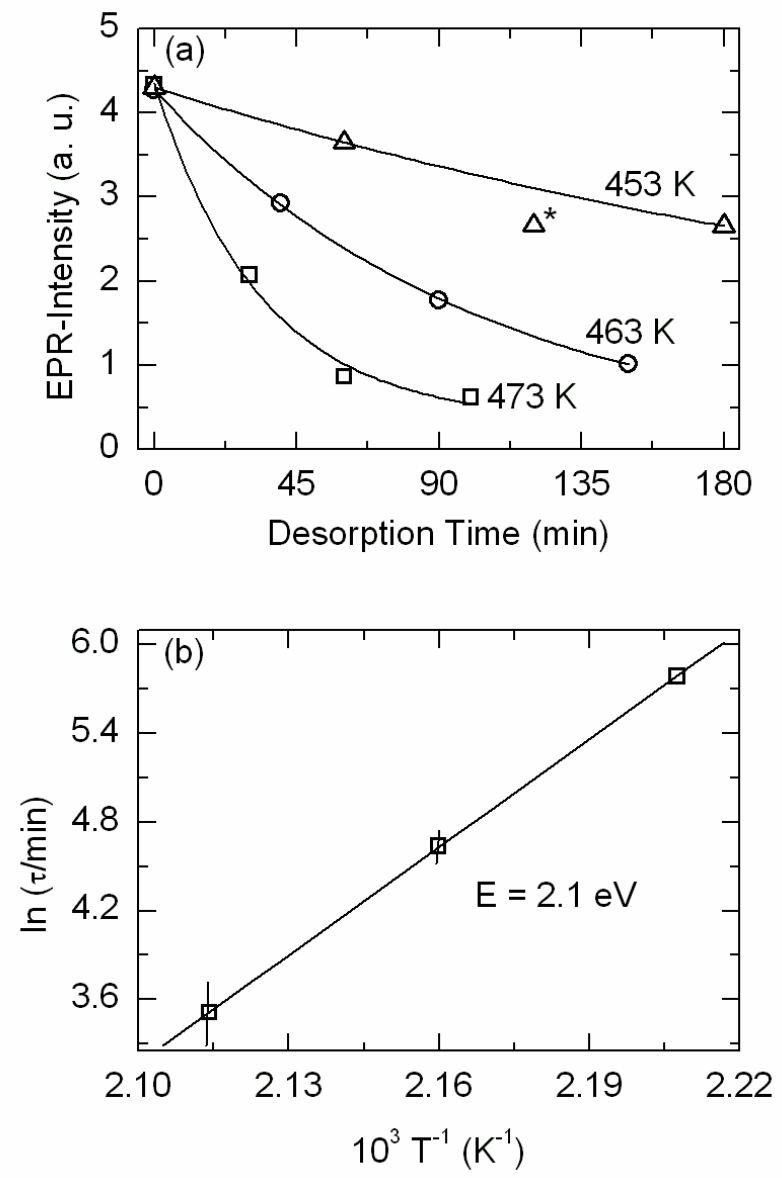

Figure 7. (a) EPR intensity as a function of desorption time at different temperatures. Data point $\triangle^{*}$ is erratic and was therefore not part of the fit. (b) Arrhenius plot of exponential decay constants $\tau$ for $\mathrm{D}_{2}$ desorption. 
zeolite framework obviously has an enormous influence on the cluster properties. This may be related to the different $\mathrm{Si} / \mathrm{Al}$ ratio as well as the different zeolite framework structure and density, as discussed above. Furthermore, the determined $\mathrm{D}_{2}$ desorption energy is clearly higher than the corresponding experimental values for desorption from a (111) single-crystal surface which center around $0.8 \mathrm{eV} .{ }^{22}$ This comparison with bulk Pt reveals another "support effect" (the absence of a zeolite) which is likely superimposed by a finite size effect of the clusters. The coordination number of surface Pt atoms on the cluster is five or six, depending on the cluster structure, which is less than that of a $\operatorname{Pt}(111)$ single crystal surface, where it is nine. Atoms with low coordination numbers have in general higher tendency to saturate their coordination spheres and tend to form stronger bonds, which is also the origin of the higher catalytic activity of nanoparticles compared to single crystal surfaces. ${ }^{14}$

H/D Isotope Exchange. More can be learned about the dynamics of the chemisorbed hydrogen in exchange experiments with deuterium, which can be monitored by EPR because the spectra of $\mathrm{H}$ and $\mathrm{D}$ exposed clusters are different, as demonstrated in Figure 1. Complete isotope exchange was achieved by adding 500 mbar of deuterium gas into an EPR tube containing about $160 \mathrm{mg}$ of hydrogen reduced $\mathrm{Pt} / \mathrm{KL}(\mathrm{CU})$ zeolite powder and leaving the sample at room temperature for 3 days. On the $\mathrm{NaY}(\mathrm{CU})$ support, $\mathrm{H} / \mathrm{D}$ isotopes exchange completely in only 30 minutes under same conditions, which is related to the lower $\mathrm{Pt}-\mathrm{H}$ binding energy on this support. Once again, there exists an immense influence of the zeolite framework on the properties of the $\mathrm{Pt}_{13}$ clusters supported in KL vs. NaY zeolite. The exchange can be accelerated by evacuation of the resulting H/D mixture in the EPR tube at half time and replacing it with $~ 500$ mbar of pure deuterium gas. Furthermore the exchange is reversible between the initial hydrogen and deuterium reduced state I as shown in Figure $1 \mathrm{a}$ and $\mathrm{b}$. 
Another interesting H/D exchange experiment is demonstrated in Figure 8. Here the exchange was carried out as reported above up to about $50 \%$, followed by desorption at $408 \mathrm{~K}$
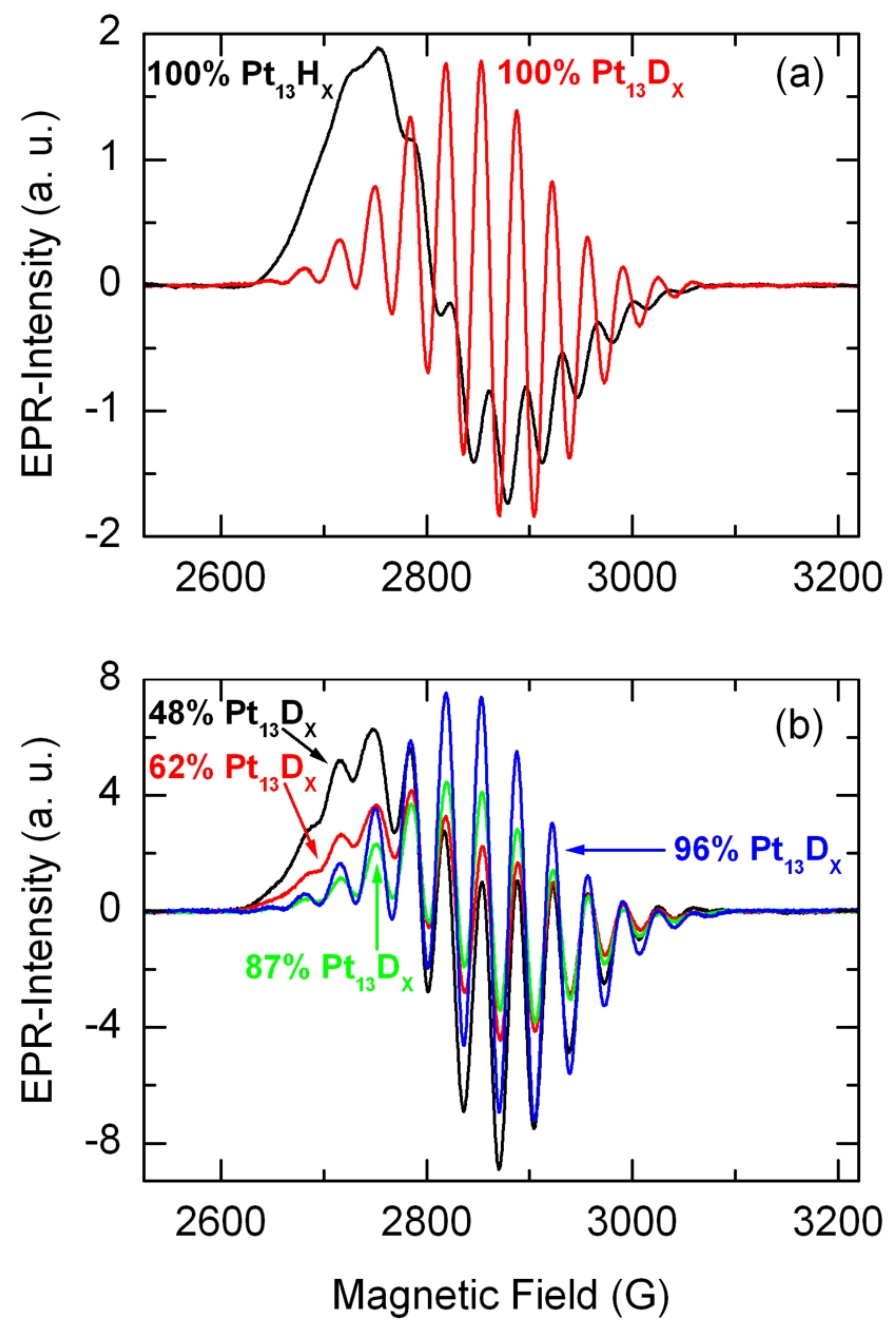

Figure 8. EPR spectra of the development of deuterium for hydrogen exchange. (a) Starting point is the initial hydrogen reduced $\mathrm{Pt}_{13}$ cluster (black line, $100 \% \mathrm{Pt}_{13} \mathrm{H}_{\mathrm{X}}$, state I) and the end point the desorbed deuterium covered $\mathrm{Pt}_{13}$ cluster (red line, $100 \% \mathrm{Pt}_{13} \mathrm{D}_{\mathrm{X}}$, state III) of the $\mathrm{H} / \mathrm{D}$ exchange experiment. (b) Isotope exchange reaction expressed in percent $\mathrm{Pt}_{13} \mathrm{D}_{\mathrm{X}}$ clusters after $\sim 1.5$ days (black line, $48 \% \mathrm{Pt}_{13} \mathrm{D}_{\mathrm{X}}$ and $52 \% \mathrm{Pt}_{13} \mathrm{H}_{\mathrm{X}}$ ), followed by desorption at $408 \mathrm{~K}$, after $30 \min$ (red line, 62\% $\mathrm{Pt}_{13} \mathrm{D}_{\mathrm{X}}$ and $38 \% \mathrm{Pt}_{13} \mathrm{H}_{\mathrm{X}}$ ), 50 min (green line, $87 \% \mathrm{Pt}_{13} \mathrm{D}_{\mathrm{X}}$ and 13\% $\mathrm{Pt}_{13} \mathrm{H}_{\mathrm{X}}$ ), and 180 min (blue line, $96 \% \mathrm{Pt}_{13} \mathrm{D}_{\mathrm{X}}$ and $4 \% \mathrm{Pt}_{13} \mathrm{H}_{\mathrm{X}}$ ). 
for different time intervals as listed in Figure 8b. As reported above, the initial reduced state I is no longer recovered after desorption, indicating that an irreversible transition of the cluster occurs on desorption. After 1.5 days, the exchange is approximately half completed as can be seen from the appearance of the $\mathrm{Pt}_{13}$ cluster multiplet demonstrated in Figure $8 \mathrm{~b}$ (black line, $48 \%$ $\left.\mathrm{Pt}_{13} \mathrm{D}_{\mathrm{X}} / 52 \% \mathrm{Pt}_{13} \mathrm{H}_{\mathrm{X}}\right)$ resulting from a superposition of hydrogen covered $\mathrm{Pt}_{13} \mathrm{H}_{\mathrm{X}}$ and deuterium covered $\mathrm{Pt}_{13} \mathrm{D}_{\mathrm{X}}$ clusters in the zeolite sample. Further desorption at $408 \mathrm{~K}$ lead to a preferential disappearance of the $\mathrm{Pt}_{13} \mathrm{H}_{\mathrm{X}}$ clusters and to a dominance of the $\mathrm{Pt}_{13} \mathrm{D}_{\mathrm{X}}$ clusters as shown in Figure $8 \mathrm{~b}$. This effect is in agreement with the above observation that the deuterated clusters are thermodynamically more stable because of their lower zero-point energy.

Because the hydrogen hyperfine splitting is not resolved it is possible to simulate the development of the H/D exchange experiment as superpositions of mole fractions of the cluster EPR spectra of the $100 \% \mathrm{Pt}_{13} \mathrm{H}_{\mathrm{X}}$ (black line) and the $100 \% \mathrm{Pt}_{13} \mathrm{D}_{\mathrm{X}}$ (red line) spectra shown in Figure 8a. The development of exchange in percent $\mathrm{Pt}_{13} \mathrm{D}_{\mathrm{X}}$ is shown in Figure $8 \mathrm{~b}$. In addition, Figure 8a indicates the small shift of the $\mathrm{Pt}_{13} \mathrm{D}_{\mathrm{X}}$ vs. the $\mathrm{Pt}_{13} \mathrm{H}_{\mathrm{X}}$ multiplet to a lower magnetic field, which corresponds to a higher $g$ value for the deuterium covered $\mathrm{Pt}_{13}$ cluster (see also Table 1).

Oxygen Adsorption. Pt is an important catalyst with applications to many reactions. The elementary catalytic step is often not well understood. It is therefore attractive to use EPR for a direct monitoring of the catalytic step as seen from the catalytic center, in particular when it is as monodisperse and well defined as the present $\mathrm{Pt}_{13}$ clusters.

It was reported previously that adsorption of carbon monoxide $(\mathrm{CO})$ on the hydrogen or deuterium covered $\mathrm{Pt}_{13}$ clusters at room temperature leads to a dramatic change in the appearance of the EPR spectrum. ${ }^{13,23}$ The cluster multiplet disappears immediately after $\mathrm{CO}$ addition, and a 
new spectrum near $g=2.0$ appears. Obviously, $\mathrm{CO}$ adsorption leads to a complete and irreversible decomposition of the cluster and, as reported by Akdogan et al., to the formation of new, binuclear $\mathrm{Pt}_{2}(\mathrm{CO})_{\mathrm{m}}(\mathrm{m}=4-5)$ species, most probably stabilized by the zeolite walls. ${ }^{23}$ Also addition of NO leads to an immediate change of the spectrum.

Usually, hydrogen and oxygen gas in the presence of platinum results in an explosive oxyhydrogen reaction. Earlier experiments have shown that $\mathrm{O}_{2}$ chemisorption on $\mathrm{Pt}(111)$ is drastically affected by a partial hydrogen overlayer due to blockage of the oxygen adsorption sites. Chemisorption occurs only on bare spots of $\mathrm{Pt}(111) \cdot{ }^{24,25}$ Deuterium covered $\mathrm{Pt}_{13}$ clusters show similar behavior for oxygen adsorption, as demonstrated in Figure 9a. No oxyhydrogen reaction was observed, and the oxygen reacts with the $\mathrm{Pt}_{13} \mathrm{D}_{\mathrm{X}}$ clusters comparatively slowly, even though the reaction is exothermic by as much as $\sim 46 \mathrm{~kJ} \mathrm{~mol}^{-1}$ (the standard reaction enthalpy minus the $\mathrm{D}_{2}$ adsorption energy). In contrast to $\mathrm{CO}$ which requires a single adsorption site, oxygen needs two neighboring sites to allow its dissociative adsorption. The deuterium coverage obviously blocks oxygen adsorption. 

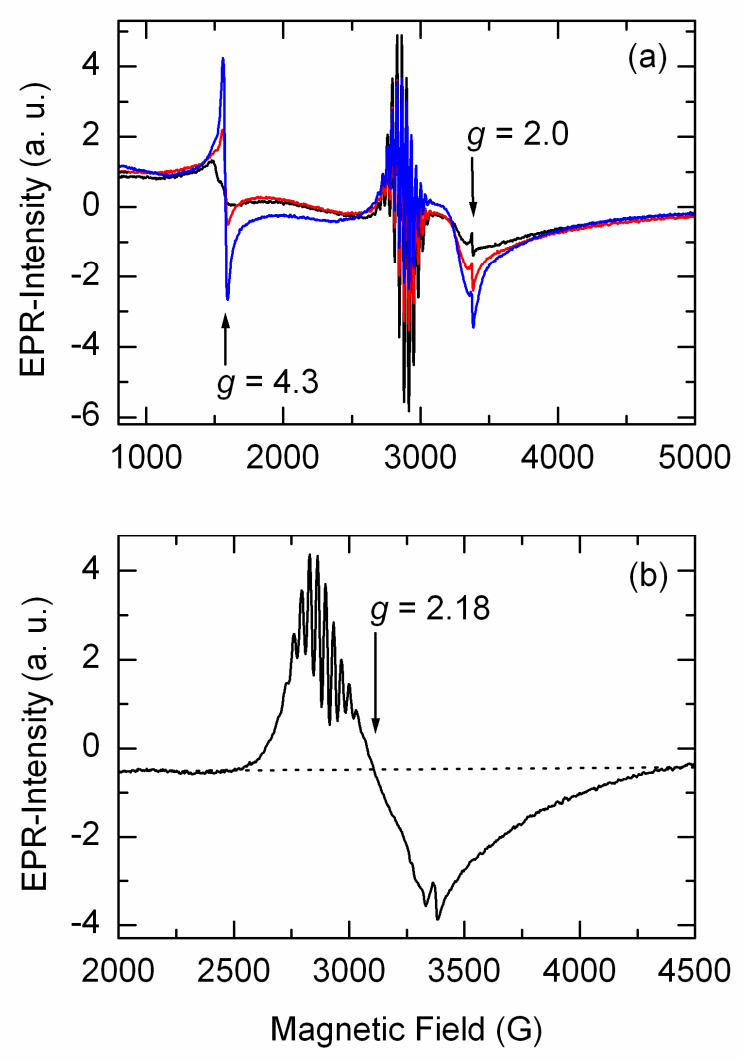

Figure 9. (a) EPR spectrum of deuterium covered $\mathrm{Pt}_{13} \mathrm{D}_{\mathrm{X}}$ clusters before oxygen adsorption (black), after exposure to $30 \mathrm{mbar} \mathrm{O}_{2}$ for $30 \mathrm{~min}$ (red), and $100 \mathrm{mbar}_{2}$ for $\sim 16 \mathrm{~h}$ (blue). (b) Enlarged EPR spectrum of $\mathrm{Pt}_{13} \mathrm{D}_{\mathrm{X}}$ clusters recorded 2 months after final oxygen adsorption (a, blue).

Besides the $\mathrm{Pt}_{13}$ cluster multiplet at $g=2.3675$ there are two other EPR signals at $g=4.3$ and $g=2.0$ due to iron impurities in the $\mathrm{KL}(\mathrm{CU})$ zeolite. The reported assignment for these signals has been as follows: isolated iron ions in cation exchange sites $(g=2.0)$, and framework iron substituting aluminum or silicon ( $g=4.3$ ), which additionally may also have a $g=2.0$ component. ${ }^{26}$ Figure 9a demonstrates that oxygen adsorption on deuterium covered $\mathrm{Pt}_{13}$ clusters leads to significant changes in the appearance of the EPR spectra. After exposure to $30 \mathrm{mbar}_{2}$ gas for 30 min the intensity of the $\mathrm{Pt}_{13} \mathrm{D}_{\mathrm{X}}$ cluster multiplet (red) clearly decreases compared to the corresponding intensity before $\mathrm{O}_{2}$ adsorption (black). However, all other parameters of the cluster 
multiplet like the $g$-factor, the intensity ratio of the splitting pattern, and the line widths remain constant. Furthermore the EPR signal of the framework iron $(g=4.3)$ shows a rising intensity possibly due to an oxidation of X-band EPR invisible $\mathrm{Fe}^{2+}$ to EPR visible $\mathrm{Fe}^{3+}$. Further exposure of the sample (about $160 \mathrm{mg} \mathrm{Pt} / \mathrm{KL}$ zeolite) to $100 \mathrm{mbar}_{2}$ gas for a longer time ( $16 \mathrm{~h}$, blue) results in an enhancement of the effects discussed above. The changes in the EPR spectra after $\mathrm{O}_{2}$ adsorption in the magnetic field range $2000 \mathrm{G}-4500 \mathrm{G}$ in Figure $9 \mathrm{a}$ are highlighted and shown enlarged in Figure 9b. The EPR spectrum presented in Figure 9b was measured approximately two months later than the blue spectrum of Figure $9 \mathrm{a}$ after final $\mathrm{O}_{2}$ exposure. Obviously there was still dynamics in the sample over this time. Superimposed on the original $\mathrm{Pt}_{13}$ multiplet and the signal of the isolated iron ions in cation exchange sites at $g=2.0$ which remains constant an unresolved second platinum species with $g=2.18$ develops (Figure $9 b$ ).

Further platinum species which are mostly concealed by the intense $\mathrm{Pt}_{13}$ cluster multiplet already existed after initial reduction (state I). After complete hydrogen desorption the $\mathrm{Pt}_{13}$ cluster multiplet disappeared and the EPR signal of this new Pt species (state V) becomes visible and remains stable as shown in other work. ${ }^{3}$ The oxygen adsorption leads to two opposite trends. The $\mathrm{Pt}_{13} \mathrm{D}_{\mathrm{X}}$ clusters get gradually EPR invisible, resulting in a decreasing EPR intensity. Meanwhile, the second platinum species with $g=2.18$ increases gradually, resulting in a rising total EPR intensity. This must mean that the secondary platinum species are also in equilibrium with similar EPR invisible species. Oxygen adsorption obviously shifts this equilibrium to the EPR visible Pt species. Deuterium covered $\mathrm{Pt}_{13}$ clusters dispersed in $\mathrm{NaY}(\mathrm{CU})$ zeolite show a completely different behavior, as reported in earlier work. ${ }^{27}$ After exposure to $\mathrm{O}_{2}$ the EPR intensity of the $\mathrm{Pt}_{13} \mathrm{D}_{\mathrm{X}}$ cluster multiplet increased rapidly. However, after exposure of the sample to $\mathrm{O}_{2}$ for $\sim 1$ day, the EPR intensity of the cluster multiplet decreased back to the original intensity before 
oxygen adsorption. The second platinum species existing also in $\mathrm{NaY}$ zeolite showed a rising intensity as well as the EPR signal of the framework iron $(g=4.3)$ like in KL zeolite. ${ }^{26}$ Once again the different zeolite frameworks influence the behavior of the $\mathrm{Pt}_{13}$ clusters.

SQUID magnetization measurements. The magnetic properties of $\mathrm{Pt}_{13}$ clusters were investigated by zero-field-cooled (ZFC) SQUID measurements of the magnetization $M(T)$. Figure 10 shows a series of quantitatively hydrogen readsorbed $\mathrm{Pt}_{13}$ cluster samples (states II-III) using a specially synthesized iron-free KL zeolite (SC) to avoid any contribution of iron and ambiguities in the interpretation of the data. ${ }^{3}$

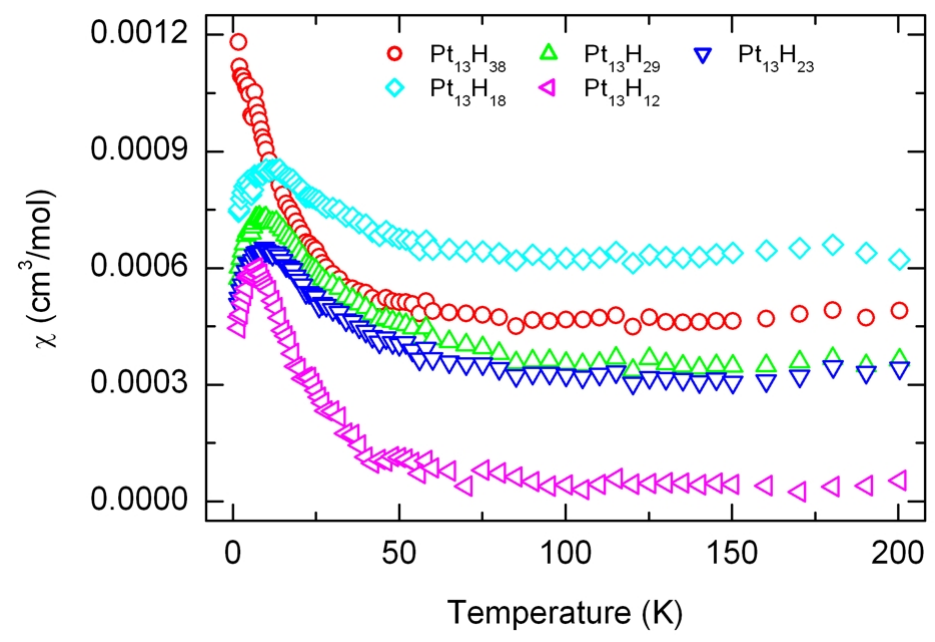

Figure 10. Susceptibility per mol Pt atoms of quantitatively hydrogen readsorbed $\mathrm{Pt}_{13}$ clusters (states II-III) as a function of temperature measured in an applied field of $2500 \mathrm{G}$ following zero-field cooling. The susceptibility has been corrected by subtraction of the contribution of the plain zeolite.

The $\mathrm{Pt}_{13}$ cluster samples show a maximum in $\chi$ at $\sim 14 \mathrm{~K}$, except for $\mathrm{Pt}_{13} \mathrm{H}_{38}$. In the temperature range above $\sim 20 \mathrm{~K}$ the $\mathrm{Pt}_{13} \mathrm{H}_{\mathrm{x}}$ clusters obey Curie-type paramagnetic behavior from which the corresponding molar Curie constants $C_{\mathrm{m}}$ and the temperature independent term $b$ are determined by fitting $\chi$ to 
$\chi=\frac{\mathrm{C}_{\mathrm{m}}}{T}+\mathrm{b}$

In non-metals, a positive temperature independent term may be ascribed to van Vleck paramagnetism which represents a coupling of the ground state with excited states. Since the term is inversely proportional to the energy difference between these states ${ }^{28}$ and the $\mathrm{Pt}_{13}$ clusters has low-lying excited states it is conceivable that the effect plays a role, but it will not be discussed further here.

Figure 11 displays the Curie constants $C_{m}$ as a function of the quantitative hydrogen coverage as presented in Figure 10.

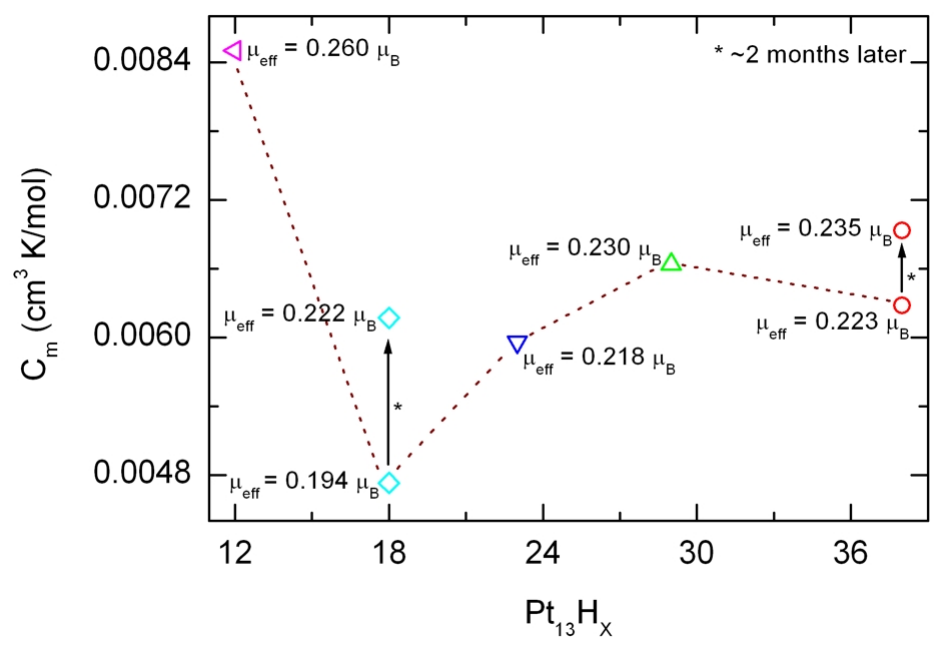

Figure 11. Curie constants $\mathrm{C}_{\mathrm{m}}$ as a function of the quantitative hydrogen covered $\mathrm{Pt}_{13}$ clusters (states II-III) and corresponding effective magnetic moments $\mu_{\text {eff }}$ per Pt atom. Asterisk: Repetition of the measurement ca. 2 months later using the same sample.

The values of $\mu_{\text {eff }}$ oscillate significantly with the amount of chemisorbed hydrogen (dotted line). This behavior is qualitatively understood based on the concept of "superatoms". Each $\mathrm{H}$ atom that binds to the $\mathrm{Pt}_{13}$ cluster surface pins one of the Pt valence d-electrons from the cluster orbital and 
engages it in a $\mathrm{Pt}_{\mathrm{n}}-\mathrm{H}$ bond. Accordingly, hydrogen chemisorption leads to a continuous variation of the electronic structure by variation of the population of cluster orbitals and to the observed oscillating behavior between higher spin and lower spin states. Furthermore, measurements of the same samples of $\mathrm{Pt}_{13} \mathrm{H}_{18}$ and $\mathrm{Pt}_{13} \mathrm{H}_{38}$ carried out two months later yield different magnetic moments, revealing clearly that the samples change with time due to equilibration over periods of weeks, while no change is observed in the EPR spectra. The hydrogen coverage of the clusters possibly changes due to spillover (the migration of hydrogen from the cluster to the support), resulting in different values for the effective magnetic moment over time. It is therefore not possible to establish a firm value for $\mu_{\text {eff }}$ as a function of coverage. Furthermore, the fact that $\mu_{\text {eff }}$ does not vary dramatically nor does it seem to go to zero at any coverage (for a closed shell configuration) is compatible with the interpretation proposed in context with Figure 3 that any nominal coverage represents the mean of a distribution of coverages so that larger fluctuations are averaged out. This also explains why the oscillation becomes smaller for higher hydrogen coverages.

Interestingly, the susceptibilities approach coverage-dependent constant values at temperatures $>100 \mathrm{~K}$. Although these are positive due to a paramagnetic offset at this low field the behavior is reminiscent of the coverage dependent giant diamagnetism that was found in higher fields. $^{12}$

\section{Conclusions}

Chemisorbed hydrogen on platinum particles is of great technical and scientific importance for catalysis and the understanding of its elementary steps. Here, we have demonstrated that EPR 
spectroscopy, in particular in combination with other methods, is a useful tool for investigating well-defined paramagnetic nanosize platinum clusters. The results provide insight into the correlation between hydrogen or deuterium coverage of the cluster and its electronic structure as well as useful information about the hydrogen desorption mechanism and energy and the different adsorption sites. The present paper is an extension of two previous studies ${ }^{10,13}$ with the main aim to put the observations on a quantitative basis, in particular as regards hydrogen coverage. Since this work concludes the series we present the conclusions in context with those of the earlier results.

- Although we can prepare monodisperse Pt nanoclusters with a well-defined size of $13 \pm 2$ atoms in zeolites ${ }^{3,10}$ there is a distribution of properties with high spin states, diamagnetic states and a minority of EPR-active spin- $-1 / 2$ states being present simultaneously ${ }^{11}$ and in equilibrium with each other. The reason for the presence of different states may relate to the microscopic heterogeneity of zeolites due to the statistical distribution (within the limits of the Loewenstein rule which excludes $\mathrm{Al}-\mathrm{O}-\mathrm{Al}$ fragment structures) of framework $\mathrm{Al}$ atoms and non-framework cations.

- The maximum number of adsorbed hydrogen atoms amounts to $2.9 \pm 0.2$ per Pt atom or $38 \pm 2$ per $\mathrm{Pt}_{13}$ cluster. $^{3}$ This is much more than conventionally assumed in catalysis where the Pt dispersion (i.e. the fraction of Pt atoms exposed to the surface) is based on a single $\mathrm{H}$ per surface Pt. The finding agrees with theoretical predictions for small clusters. ${ }^{4,5}$

- The deuterium desorption energy from the Pt clusters is $2.1 \pm 0.2 \mathrm{eV}$ in zeolite $\mathrm{KL}(\mathrm{Si} / \mathrm{Al}=$ 3.0) compared to $1.36 \mathrm{eV}$ in zeolite $\mathrm{NaY}(\mathrm{Si} / \mathrm{Al}=2.3)^{14}$ and $0.8 \mathrm{eV}$ from a single crystal 
Pt surface. ${ }^{22}$ This dependence on the framework structure demonstrates a clear support effect, and in comparison with the bulk possibly also a quantum size effect.

- The geometric structure depends on the amount of chemisorbed hydrogen and on the preparation history of the sample, as shown by EXAFS, ${ }^{3}$ EPR and HYSCORE spectroscopy. When hyperfine couplings are resolved the Pt atoms of a cluster are always equivalent and show identical isotropic values within the experimental resolution, but there is a small anisotropy that depends on coverage. Three sets of proton hyperfine couplings were observed. In most states of the sample there is a single set of equivalent protons, but on readsorption after complete hydrogen desorption two different sets are seen.

- The small axial anisotropy of the $g$ tensor derived from an EPR spectrum reflects the symmetry of the orbitals containing the unpaired electrons. The occupancy of these cluster (or pseudoatom) ${ }^{9}$ orbitals is a strong function of hydrogen coverage, since each additional $\mathrm{H}$ binds one of the electrons in the highest occupied orbital. Despite this small $g$ anisotropy the equivalence of the hyperfine couplings points to an almost perfect high symmetry, possibly near icosahedral or cuboctahedral (remembering that open shell systems often undergo a Jahn-Teller distortion). Hoevever, after the initial reduction and after complete desorption structureless broad lines are present which likely represent low symmetry species. Such structural changes are supported by theoretical predictions.

- The spin state of transition metal ions depends on the oxidation state. In analogy, the magnetic properties of the $\mathrm{Pt}_{13} \mathrm{H}_{\mathrm{x}}$ clusters depend also on the occupancy of the cluster orbitals which can be varied by the hydrogen coverage. This is also seen in the SQUID magnetization measurements. While the EPR spectra reveal incomplete reversibility only 
between the initial reduced state I and state II, and between the fully desorbed state V and state III, the SQUID magnetization values show slow equilibration which is not seen in the EPR active fraction.

- On exposure to $\mathrm{CO}$ the $\mathrm{Pt}_{13}$ clusters react by fragmentation to binuclear $\mathrm{Pt}$ carbonyls. ${ }^{23}$ However, the reaction of $\mathrm{O}_{2}$ with $\mathrm{Pt}_{13} \mathrm{D}_{\mathrm{x}}$ is extremely sluggish although thermodynamically clearly favored. The explanation probably lies in the number of adsorption sites: CO binds atop the Pt atom and needs a single site. Atop sites are not the favored adsorption sites for $\mathrm{H}(\mathrm{D}) . \mathrm{O}_{2}$ needs two neighboring sites to bind and dissociate. It thus has to compete with $\mathrm{H}(\mathrm{D})$ which also binds preferentially to edge sites. Furthermore, if an icosahedral $\mathrm{Pt}_{13}$ cluster has a free edge site across two Pt atoms the remaining 8 edges which start at these atoms may be occupied by one H(D) each. These hydrogen(deuterium) atoms are hydridic in character and deplete the electron density on Pt, which diminishes its potential to form a bond to the electronegative oxygen.

\section{Corresponding Author}

* To whom correspondence should be addressed.

E-mail: e.roduner@ipc.uni-stuttgart.de. Fax: (+49) 711-685-64495

\section{Acknowledgements}

Chemie Uetikon AG in Switzerland and to Süd Chemie AG in Germany are greatly acknowledged for providing KL zeolite for our research. We are grateful to Michal Gorski for his support within a research project in our group and we thank Johannes Kollmer for helping us create the 3D images displayed in Figure 2. 


\section{References}

(1) Sachtler, W. M. H. Zeolite-Supported Transition Metal Catalysts by Design.

Catal. Today 1992, 15, 419-429.

(2) Teo, B. K.; Sloane, N. J. A. Magic Numbers in Polygonal and Polyhedral Clusters. Inorg. Chem. 1985, 24, 4545-4558.

(3) Jensen, C.; Buck, D.; Dilger, H.; Bauer, M.; Phillipp, F.; Roduner, E. Maximum Hydrogen Chemisorption on KL Zeolite Supported Pt Clusters. Chem. Commun. 2013, 49, 588-590.

(4) Mager-Maury, C.; Bonnard, G.; Chizallet, C.; Sautet, P.; Raybaud, P. H2-Induced Reconstruction of Supported Pt Clusters: Metal-Support Interaction Versus Surface Hydride. ChemCatChem, 2011, 3, 200-207.

(5) Chen, L.; Zhou, C.; Wu, J.; Cheng, H. Hydrogen Adsorption and Desorption on the Pt and Pd Subnano Clusters - a Review. Front. Phys. China, 2009, 4, 356-366.

(6) Watari, N.; Ohnishi, S., Electronic Structure of H Adsorbed on $\mathrm{Pt}_{13}$ Clusters. J. Chem. Phys. 1997, 106, 7531-7540.

(7) Watari, N.; Ohnishi, S., Atomic and Electronic Structures of $\mathrm{Pd}_{13}$ and $\mathrm{Pt}_{13}$ Clusters. Phys. Rev. B, 1998, 58, 1665-1677.

(8) de Heer, W. A., The Physics of Simple Metal Clusters: Experimental Aspects and Simple models. Rev. Mod. Phys. 1993, 65, 611-676. 
(9) Roduner, E., Size Matters: Why Nanomaterials Are Different. Chem. Soc. Rev. 2006, 35, 583-592.

(10) Liu, X. et al., Structure and Magnetization of Small Monodisperse Platinum Clusters. Phys. Rev. Lett. 2006, 97, 253401; 2009, 102, 049902.

(11) Bartolomé, J.; Bartolomé, L.; García, L. M.; Roduner, E.; Akdogan, Y.; Wilhelm, F.; Rogalev, A. Magnetization of $\mathrm{Pt}_{13}$ Clusters Supported in a NaY Zeolite: A XANES and XMCD Study. Phys. Rev. B 2009, 80, 014404.

(12) Roduner, E.; Jensen, C.; van Slageren, J.; Rakoczy, R. A.; Larlus, O.; Hunger, M., Anomalous Diamagnetic Susceptibility in 13-Atom Pt Nanocluster Superatoms, in preparation.

(13) Schmauke, T.; Eichel, R. A.; Schweiger, A.; Roduner, E. Electron Paramagnetic Resonance Studies of a Platinum Cluster in Linde L and Faujasite Zeolites. Phys. Chem. Chem. Phys. 2003, 5, 3076-3084.

(14) Liu, X.; Dilger, H.; Eichel, R. A.; Kunstmann, J.; Roduner, E. A Small Paramagnetic Platinum Cluster in an NaY Zeolite: Characterization and Hydrogen Adsorption and Desorption. J. Phys. Chem. B, 2006, 110, 2013-2023.

(15) Blundell, Stephen, Magnetism in Condensed Matter, Oxford Master Series in Condensed Matter Physics, Oxford University Press, Oxford, 2001.

(16) Dyrek, K.; Che, M. EPR as a Tool to Investigate the Transition Metal Chemistry on Oxide Surfaces. Chem. Rev. 1997, 97, 305-331. 
(17) Ji, Y.; van der Eerden, A. M. J.; Koot, V.; Kooyman, P. J.; Meeldijk, J. D.; Weckhuysen, B. M.; Koningsberger, D. C. Influence of Support Ionicity on the Hydrogen Chemisorption of Pt Particles Dispersed in Y Zeolite: Consequences for Pt Particle Size Determination Using the H/M Method. J. Catal. 2005, 234, 376-384.

(18) Breck, D. W. Zeolite Molecular Sieves, Wiley, New York, 1974.

(19) Oudenhuijzen, M. K.; Bitter, J. H.; Koningsberger, D. C. The Nature of the Pt-H Bonding for Strongly and Weakly Bonded Hydrogen on Platinum. A XAFS Spectroscopy Study of the Pt-H Antibonding Shaperesonance and Pt-H EXAFS.

J. Phys. Chem. B 2001, 105, 4616-4622.

(20) Höfer, P.; Grupp, A.; Nebenführ, H.; Mehring, M. Hyperfine Sublevel Correlation (HYSCORE) Spectroscopy: a 2D ESR Investigation of the Squaric Acid Radical. Chem. Phys. Lett. 1986, 132, 279-282.

(21) Pöppl, A.; Kevan, L. A Practical Strategy for Determination of Proton Hyperfine Interaction Parameters in Paramagnetic Transition Metal Ion Complexes by TwoDimensional HYSCORE Electron Spin Resonance Spectroscopy in Disordered Systems. J. Phys. Chem. 1996, 100, 3387-3394.

(22) Badescu, S. C.; Salo, P.; Ala-Nissila, T.; Ying, S. C.; Jacobi, K.; Wang, Y.; Bedürftig, K.; Ertl, G. Energetics and Vibrational States for Hydrogen on Pt(111). Phys. Rev. Lett. 2002, $88,136101$. 
(23) Akdogan, Y.; Anantharaman, S.; Liu, X.; Lahiri, G. K.; Bertagnolli, H.; Roduner, E. Reconstruction of $\mathrm{Pt}_{13}$ Clusters into $\mathrm{Pt}_{2}(\mathrm{CO})_{m}$ on $\mathrm{CO}$ Addition in NaY Zeolite.

J. Phys. Chem. C, 2009, 113, 2352-2359.

(24) Gustafsson, K.; Andersson, S., Leakage of $\mathrm{O}_{2}$ Precursor Molecules from Inert Hydrogen Islands on a Pt(111) Surface. Phys. Rev. Lett. 2006, 97, 076101.

(25) MacNaughton, J. B.; Näslund, L.; Anniyev, T.; Ogasawara, H.; Nilsson, A. Peroxide-Like Intermediate Observed at Hydrogen Rich Condition on Pt(111) After Interaction with Oxygen. Phys. Chem. Chem. Phys. 2010, 12, 5712-5716.

(26) Goldfarb, D.; Bernardo, M.; Strohmaier, K. G.; Vaughan, D. E. W.; Thomann, H. Characterization of Iron in Zeolites by X-band and Q-Band ESR, Pulsed ESR, and UVVisible Spectroscopies. J. Am. Chem. Soc. 1994, 116, 6344-6353.

(27) Liu, X. Pt clusters in NaY zeolite studied by EPR, ENDOR, and SQUID measurements, and by DFT calculations; Dissertation 2006; pp 98-102.

(28) Lueken, H. Magnetochemie: Eine Einführung in Theorie und Anwendung, Teubner, Stuttgart, Leipzig, 1999.

\section{Table of content graphic:}
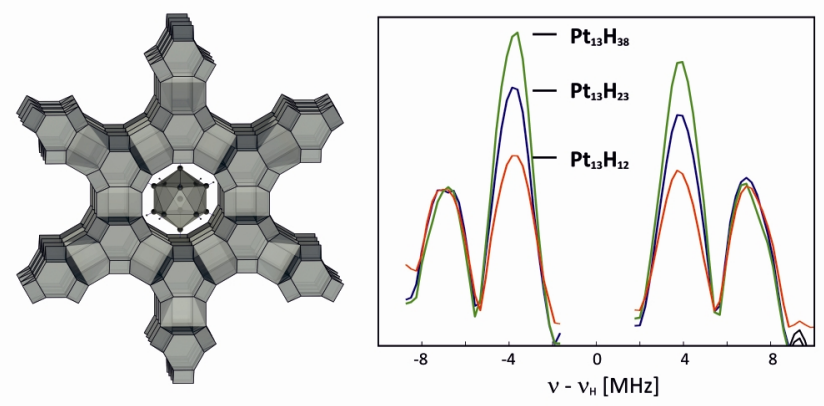